\title{
Expert consensus on perioperative immunotherapy for local advanced non-small cell lung cancer
}

\begin{abstract}
Bin Qiu ${ }^{1}$, Kaican Cai ${ }^{2}$, Chun Chen ${ }^{3}$, Jun Chen ${ }^{4}$, Ke-Neng Chen ${ }^{5}$, Qi-Xun Chen ${ }^{6,7}$, Chao Cheng ${ }^{8}$, Tian-Yang Dai ${ }^{9}$, Junqiang Fan ${ }^{10}$, Zhaohui Fan ${ }^{11}$, Jian $\mathrm{Hu}^{12}$, Wei-Dong Hu ${ }^{13}$, Yun-Chao Huang ${ }^{14}$, Ge-Ning Jiang ${ }^{15}$, Jie Jiang ${ }^{16}$, Tao Jiang ${ }^{17}$, Wen-Jie Jiao ${ }^{18}$, He-Cheng Li $^{19}$, Qiang Li $^{20}$, Yong-De Liao ${ }^{21}$, Hong-Xu Liu ${ }^{22}$, Jun-Feng Liu ${ }^{23}$, Lunxu Liu ${ }^{24}$, Yang Liu ${ }^{25}$, Hao Long ${ }^{26}$, Qing-Quan Luo ${ }^{27}$, Hai-Tao Ma ${ }^{28}$, Nai-Quan Mao ${ }^{29}$, Xiao-Jie Pan ${ }^{30}$, Fengwei Tan ${ }^{1}$, Li-Jie Tan ${ }^{31}$, Hui Tian ${ }^{32}$, Dong Wang ${ }^{33}$, Wen-Xiang Wang ${ }^{34}$, Li $\mathrm{Wei}^{35}$, Nan $\mathrm{Wu}^{36}$, Qing-Chen Wu ${ }^{37}$, Jiaqing Xiang ${ }^{38}$, Shi-Dong Xu ${ }^{39}$, Lin Yang ${ }^{40}$, Hao Zhang ${ }^{41}$, Lanjun Zhang $^{42}$, Peng Zhang ${ }^{15}$, Yi Zhang ${ }^{43}$, Zhenfa Zhang ${ }^{44}$, Kunshou Zhu ${ }^{45}$, Yuming Zhu ${ }^{46}$, Sang-Won Um ${ }^{47}$, In-Jae $\mathrm{Oh}^{48}$, Yusuke Tomita ${ }^{49}$, Satoshi Watanabe ${ }^{50}$, Takeo Nakada ${ }^{51}$, Nobuhiko Seki ${ }^{52}$, Toyoaki Hida ${ }^{53}$, Shinji Sasada $^{54}$, Junji Uchino ${ }^{55}$, Haruhiko Sugimura ${ }^{56}$, Said Dermime ${ }^{57}$, Federico Cappuzzo ${ }^{58}$, Stefania Rizzo ${ }^{59}$, William Chi-Shing Cho ${ }^{60}$, Pierfilippo Crucitti ${ }^{61}$, Filippo Longo ${ }^{61}$, Kye Young Lee ${ }^{62}$, Dirk De Ruysscher ${ }^{63}$, Ben G. L. Vanneste ${ }^{63}$, Muhammad Furqan ${ }^{64}$, Jessica C. Sieren ${ }^{65}$, Sai Yendamuri ${ }^{66}$, Kenneth W. Merrell ${ }^{67}$, Julian R. Molina ${ }^{68}$, Giulio Metro ${ }^{69}$, Raffaele Califano ${ }^{70}$, Stefano Bongiolatti ${ }^{71}$, Mariano Provencio ${ }^{72}$, Paul Hofman ${ }^{73}$, Shugeng Gao ${ }^{1}$, Jie $\mathrm{He}^{1}$
\end{abstract}

${ }^{1}$ Department of Thoracic Surgery, National Cancer Center/National Clinical Research Center for Cancer/Cancer Hospital, Chinese Academy of Medical Sciences and Peking Union Medical College, Beijing, China; ${ }^{2}$ Department of Thoracic Surgery, Nanfang Hospital, Southern Medical University, Guangzhou, China; ${ }^{3}$ Department of Thoracic Surgery, Fujian Medical University Union Hospital, Fuzhou, China; ${ }^{4}$ Tianjin Key Laboratory of Lung Cancer Metastasis and Tumor Microenvironment, Lung Cancer Institute, Tianjin Medical University General Hospital, Tianjin, China; ${ }^{5}$ Department of Thoracic Surgery, Key Laboratory of Carcinogenesis and Translational Research (Ministry of Education), Peking University Cancer Hospital \& Institute, Beijing, China; ${ }^{6}$ Department of Thoracic Surgery, Cancer Hospital of University of Chinese Academy of Sciences (Zhejiang Cancer Hospital), Hangzhou, China; ${ }^{7}$ Institute of Cancer and Basic Medicine (IBMC), Chinese Academy of Science, Hangzhou, China; ${ }^{8}$ Department of Thoracic Surgery, the First Affiliated Hospital, Sun Yat-sen University, Guangzhou, China; ${ }^{9}$ Department of Thoracic Surgery, The Affiliated Hospital of Southwest Medical University, Luzhou, China; ${ }^{10}$ Department of Thoracic Surgery, Second Affiliated Hospital of Zhejiang University, School of Medicine, Hangzhou, China; ${ }^{11}$ Department of Thoracic Surgery, Jiangsu Cancer Hospital (Nanjing Medical University Affiliated Cancer Hospital) and Jiangsu Institute of Cancer Research, Nanjing, China; ${ }^{12}$ Department of Thoracic Surgery, The First Affiliated Hospital, College of Medicine, Zhejiang University, Hangzhou, China; ${ }^{13}$ Department of Thoracic Surgery, Zhongnan Hospital of Wuhan University, Wuhan, China; ${ }^{14}$ Department of Thoracic Surgery, Yunnan Cancer Hospital, Kunming, China; ${ }^{15}$ Department of Thoracic Surgery, Shanghai Pulmonary Hospital, Tongji University School of Medicine, Shanghai, China; ${ }^{16}$ Department of Thoracic Surgery, The First Affiliated Hospital of Xiamen University, Xiamen, China; ${ }^{17}$ Department of Thoracic Surgery, Tangdu Hospital, Fourth Military Medical University, Xi'an, China; ${ }^{18}$ Department of Thoracic Surgery, The Affiliated Hospital of Qingdao University, Qingdao, China; ${ }^{19}$ Department of Thoracic Surgery, Ruijin Hospital, Shanghai Jiao Tong University School of Medicine, Shanghai, China; ${ }^{20}$ Department of Thoracic Surgery, Sichuan Cancer Hospital and Institute, Sichuan Cancer Center, School of Medicine, University of Electronic Science and Technology of China, Chengdu, China; ${ }^{21}$ Union Hospital, Tongji Medical College, Huazhong University of Science and Technology, Wuhan, China; ${ }^{22}$ Department of Thoracic Surgery, Cancer Hospital of China Medical University, Liaoning Cancer Hospital \& Institute, Shenyang, China; ${ }^{23}$ Department of Thoracic Surgery, The Fourth Hospital of Hebei Medical University, Shijiazhuang, China; ${ }^{24}$ Department of Thoracic Surgery, West China Hospital, Sichuan University, Chengdu, China; ${ }^{25}$ Department of Thoracic Surgery, Chinese PLA General Hospital, Beijing, China; ${ }^{26}$ Department of Thoracic Surgery, State Key Laboratory of Oncology in South China, Collaborative Innovation Center for Cancer Medicine, Sun Yat-sen University Cancer Center, Guangzhou, China; ${ }^{27}$ Shanghai Lung Cancer Center, Shanghai Chest Hospital, Shanghai Jiao Tong University, Shanghai, China; ${ }^{28}$ Department of Thoracic Surgery, The First Affiliated Hospital of Soochow University, Suzhou, China; ${ }^{29}$ Department of Thoracic Surgery, Tumor Hospital Affiliated to Guangxi Medical University, Nanning, China; ${ }^{30}$ Department of Thoracic Surgery, Fujian Provincial Hospital, Fuzhou, China; ${ }^{31}$ Department of Thoracic Surgery, Zhongshan Hospital, Fudan University, Shanghai, China; ${ }^{32}$ Department of Thoracic Surgery, Qilu Hospital, Cheeloo College of Medicine, Shandong University, Jinan, China; ${ }^{33}$ Department of Cardiothoracic Surgery, Affiliated Taikang Xianlin Drum Tower Hospital, Medical School of Nanjing University, Nanjing, China; ${ }^{34}$ Department of Thoracic Surgery II, The Affiliated Cancer Hospital of Xiangya School of Medicine, Central South University/Hunan Cancer Hospital, Changsha, China; ${ }^{35}$ Henan Provincial People's Hospital, Zhengzhou, China; ${ }^{36}$ Key Laboratory of Carcinogenesis 
and Translational Research (Ministry of Education), Department of Thoracic Surgery II, Peking University Cancer Hospital \& Institute, Beijing, China; ${ }^{37}$ Department of Cardiothoracic Surgery, The First Affiliated Hospital of Chongqing Medical University, Chongqing, China; ${ }^{38}$ Department of Thoracic Surgery, Fudan University Shanghai Cancer Center, Shanghai, China; ${ }^{39}$ Department of Thoracic Surgery, Harbin Medical University Cancer Hospital, Harbin, China; ${ }^{40}$ Department of Thoracic Surgery, Shenzhen People's Hospital, 2nd Clinical Medical College of Jinan University, Shenzhen, China; ${ }^{41}$ Department of Thoracic Cardiovascular Surgery, The Affiliated Hospital of Xuzhou Medical University, Xuzhou, China; ${ }^{42}$ Department of Thoracic Surgery, State Key Laboratory of Oncology in South China, Collaborative Innovation Center for Cancer Medicine, Sun Yat-sen University Cancer Center, Guangdong Esophageal Cancer Institute, Guangzhou, China; ${ }^{43}$ Department of Thoracic Surgery, Xuanwu Hospital of Capital Medical University, Beijing, China; ${ }^{44}$ Department of Lung Cancer, Tianjin Medical University Cancer Institute \& Hospital, Tianjin, China; ${ }^{45}$ Department of Thoracic Surgery, Fujian Cancer Hospital and Fujian Medical University Cancer Hospital, Fuzhou, Fujian, China; ${ }^{46}$ Department of Thoracic Surgery, Shanghai Pulmonary Hospital, School of Medicine, Tongji University, Shanghai, China; ${ }^{47}$ Division of Pulmonary and Critical Care Medicine, Department of Medicine, Samsung Medical Center, Sungkyunkwan University School of Medicine, Seoul, Korea; ${ }^{48}$ Department of Internal Medicine, Chonnam National University Medical School and Hwasun Hospital, Jeonnam, Korea; ${ }^{49}$ Department of Respiratory Medicine, Graduate School of Medical Sciences, Kumamoto University, Kumamoto, Japan; ${ }^{50}$ Department of Respiratory Medicine and Infectious Diseases, Niigata University Graduate School of Medical and Dental Sciences, Niigata, Japan; ${ }^{51}$ Department of Thoracic Surgery, Aichi Cancer Center Hospital, Nagoya, Japan; ${ }^{52}$ Division of Medical Oncology, Department of Internal Medicine, Teikyo University School of Medicine, Tokyo, Japan; ${ }^{53}$ Lung Cancer Center, Central Japan International Medical Center, Gifu, Japan; ${ }^{54}$ Department of Respiratory Medicine, Tokyo Saiseikai Central Hospital, Tokyo, Japan; ${ }^{55}$ Department of Pulmonary Medicine, Graduate School of Medical Science, Kyoto Prefectural University of Medicine, Kyoto, Japan; ${ }^{56}$ Department of Tumor Pathology, Hamamatsu University School of Medicine, Hamamatsu, Shizuoka, Japan; ${ }^{57}$ Department of Medical Oncology and Translational Research Institute, National Center for Cancer Care and Research, Hamad Medical Corporation, Doha, Qatar; ${ }^{58}$ Division of Medical Oncology 2, IRCCS Regina Elena National Cancer Institute, Rome, Italy; ${ }^{59}$ Imaging Institute of the Southern Switzerland (IIMSI), Ente Ospedaliero Cantonale (EOC), Università della Svizzera Italiana, Lugano, Switzerland; ${ }^{60}$ Department of Clinical Oncology, Queen Elizabeth Hospital, Hong Kong, China; ${ }^{61}$ Department of Thoracic Surgery, University Campus Bio-Medico, Rome, Italy; ${ }^{62}$ Precision Medicine Lung Cancer Center, Konkuk University Medical Center, Seoul, Korea; ${ }^{63}$ Department of Radiation Oncology, MAASTRO Clinic, GROW School for Oncology and Developmental Biology, Maastricht University Medical Centre, Maastricht, The Netherlands; ${ }^{64}$ Division of Hematology, Oncology and Blood and Marrow Transplantation, Department of Internal Medicine, University of Iowa, Iowa City, IA, USA; ${ }^{65}$ Department of Radiology and Biomedical Engineering, University of Iowa, Iowa City, IA, USA; ${ }^{66}$ Department of Thoracic Surgery, Roswell Park Cancer Institute, Buffalo, NY, USA; ${ }^{67}$ Department of Radiation Oncology, Mayo Clinic, Rochester, MN, USA; ${ }^{68}$ Division of Medical Oncology, Mayo Clinic, Rochester, MN, USA; ${ }^{69}$ Medical Oncology, Santa Maria della Misericordia Hospital, Azienda Ospedaliera di Perugia, Perugia, Italy; ${ }^{70}$ Department of Medical Oncology, The Christie NHS Foundation Trust and Division of Cancer Sciences, The University of Manchester, Manchester, UK; ${ }^{71}$ Thoracic Surgery Unit, Careggi University Hospital, Florence, Italy; ${ }^{72}$ Medical Oncology Department, Hospital Universitario Puerta de Hierro Majadahonda, Madrid, Spain; ${ }^{73}$ Laboratory of Clinical and Experimental Pathology, FHU OncoAge, Pasteur Hospital, BB-003300025, CHU Nice, Université Côte d'Azur, Nice, France

Correspondence to: Shugeng Gao, MD. Department of Thoracic Surgery, National Cancer Center/National Clinical Research Center for Cancer/ Cancer Hospital, Chinese Academy of Medical Sciences and Peking Union Medical College, Beijing, China. Email: gaoshugeng@vip.sina.com.

Submitted Jun 07, 2021. Accepted for publication Sep 18, 2021.

doi: $10.21037 /$ tlcr-21-634

View this article at: https://dx.doi.org/10.21037/tlcr-21-634

\section{Background}

The treatment of lung cancer is one of the major challenges in the field of oncology. According to statistics from the National Cancer Center of China in 2015, lung cancer has the highest incidence and mortality, with 733,300 new cases and 610,200 deaths across the country (1). About $85 \%$ of lung cancers are non-small cell lung cancer (NSCLC), of which $30 \%$ to $40 \%$ are considered resectable tumors, including most stage I-II and a small portion of stage IIIA tumors (2).

Very early-stage NSCLC (IA) can be cured by surgery. However, more than $50 \%$ of NSCLC patients who undergo surgical treatment will relapse or metastasize within 5 years. Even if there is no lymph node metastasis and the primary 
tumor is less than $1 \mathrm{~cm}$, nearly $8 \%$ of patients still die of the disease within 5 years after anatomical resection $(3,4)$. To improve the prognosis of resectable NSCLC, adjuvant and neoadjuvant chemotherapy has been widely used as the perioperative treatment. Neoadjuvant chemotherapy can increase the chance of radical resection by reducing tumor volume, eliminating micrometastasis, and reducing tumor recurrence risk. However, the 5-year survival rate of patients receiving either neoadjuvant or adjuvant chemotherapy only improves by approximately $5 \%(5,6)$. The use of the neoadjuvant approach is not common except in the setting of resectable stage IIIA NSCLC and does not yield particularly different survival outcomes. Compared to the adjuvant approach, neoadjuvant therapy can help eliminate micrometastases early on; however, concern for the progression of disease while neoadjuvant therapy is ongoing has inclined the surgical oncology community to operate on tumors early on and rely on systemic therapy in the adjuvant setting.

After the emergence of immune checkpoint inhibitors (ICIs) [programmed cell death protein 1/programmed cell death-ligand 1 (PD-1/L1) antibody and cytotoxic T-lymphocyte-associated protein 4 (CTLA-4) antibody], the treatment model for advanced NSCLC has completely changed, and the progression-free survival (PFS) and the overall survival (OS) of patients have been significantly improved. Immunotherapy has moved from the secondline treatment of advanced NSCLC to the first line, and to the consolidation therapy of locally advanced NSCLC patients who receive chemoradiation. Its application has now expanded into the neoadjuvant and adjuvant setting for resectable NSCLC. Immunotherapy use in the neoadjuvant setting is critical because, if the outcome of neoadjuvant therapy improves, then resection of NSCLC can offer cure to a higher number of patients. ICIs have already been shown to provide patients with better survival benefits in the neoadjuvant treatment of melanoma and glioma $(7,8)$. In some phase II clinical trials of resectable NSCLC, the major pathological response (MPR) rate of patients receiving neoadjuvant immunotherapy was as high as $45 \%$ (9).

In order to reduce clinical staging, increase surgical resection rate, reduce tumor burden, decrease postoperative recurrence, prolong survival, and ultimately achieve the goal of benefiting more patients, a series of clinical trials of perioperative immunotherapy was conducted in recent years. To better guide Chinese thoracic surgeons in the neoadjuvant immunotherapy of NSCLC, the "Expert consensus on neoadjuvant immunotherapy for non-small cell lung cancer" was published last year (10). However, more recent investigations have employed different strategies of perioperative immunotherapy. To update the current evidence and standardize clinical practice, wellknown thoracic surgeons in China participated in an indepth discussion on controversial issues and topics du jour, forming the 2021 "Expert consensus on perioperative immunotherapy for NSCLC".

\section{Consensus 1: overview of perioperative immunotherapy for NSCLC patients}

* Although anatomic resection seems curative, most postoperative NSCLC patients are at risk of recurrence or metastasis, and the risk increases with the disease stage.

- Distant metastasis is the most common mode of recurrence after anatomic resection of NSCLC. Adjuvant or neoadjuvant therapy should focus on the prevention of postoperative metastasis.

* Both neoadjuvant or adjuvant chemotherapy for NSCLC has improved the OS rate of patients by about $5 \%$; there is no significant difference between the two options, but the efficacy has reached a plateau.

* Different from perioperative chemotherapy, ICIs provide a new perspective on the perioperative treatment of NSCLC.

* ICIs may have a long-lasting effect and are suitable for perioperative treatment of operable NSCLC.

* Multidisciplinary team discussion is helpful in developing the application of perioperative immunotherapy.

Goldstraw et al. (11) reported a study of over 100,000 patients from 46 research centers in 19 countries and showed that the majority of NSCLC patients undergoing complete resection have recurrence and metastasis after surgery. Many previous studies have shown that, compared with local recurrence, distant metastasis is the most common mode of postoperative relapse (12-14). The recurrence risk increases with the tumor-node-metastasis (TNM) clinical stage and significantly reduces the OS of patients. In order to further improve outcomes of early-stage NSCLC patients, the focus of perioperative treatment should be on how to prevent or treat postoperative metastasis.

Current studies have shown that perioperative neoadjuvant and adjuvant chemotherapy can improve the prognosis of NSCLC patients. However, the survival benefit of chemotherapy has reached a plateau at about $5 \%(6,7)$, and developing an approach to improving the 
efficacy of perioperative treatment and circumventing this bottleneck has become a focus of recent clinical research.

Compared with traditional chemotherapy, immunotherapy has repeatedly achieved good results in advanced NSCLC, improving the efficacy, reducing the toxicity, and providing a new perspective for the perioperative treatment of NSCLC (15-17). Patients with malignant tumor are generally in a state of immunosuppression. After surgery, due to trauma and tumor antigen loss, the immunosuppressive state may be more severe, and a "window period" of immune deficiency may even be present (18). Therefore, the use of immunotherapy to activate the immune system before surgery may enable the patient to persist through the state of immunodeficiency and obtain long-term survival benefits (19). In addition, immunotherapy may better activate the lymphocytes with the native structure of blood vessels and regional lymph nodes prior to resection, leading to more effective tumor killing (20). At the molecular pathology level, tumor tissues after neoadjuvant immunotherapy often contain a large number of lymphocytes, some of which regulate $\mathrm{T}$ cells and CD8+ T cells, and can be used as markers to analyze therapy response (21). After immunotherapy, individuals who produce higher levels of CD8+ T cells in blood and tissues may have a better prognosis (22).

\section{Consensus 2: patient selection for neoadjuvant immunotherapy}

* All patients who are ready to receive neoadjuvant immunotherapy need to be pathologically diagnosed with NSCLC with next-generation sequencing (NGS) before treatment.

* Neoadjuvant immunotherapy has no clear predictive markers, but it must be used with caution when other mutations are identified, such as epidermal growth factor receptor (EGFR)-sensitive mutations or anaplastic lymphoma kinase (ALK) fusion alteration.

* Patients with resectable NSCLC may consider neoadjuvant immunotherapy combined with platinum doublet chemotherapy or neoadjuvant single-agent immunotherapy before surgery.

* For unresectable locally advanced NSCLC, immunotherapy plus (or without) chemotherapy induction can be considered, and the possibility of radical resection can be reassessed after downstaging.

In Asia, 40-50\% of lung adenocarcinoma patients have an epidermal EGFR gene-activating mutation. Therefore, exploring the relationship between driver gene status and neoadjuvant immunotherapy is of great significance in an Asian population. In the subgroup analysis of clinical trials, including the Checkmate 057, Keynote-010, and OAKE trials, advanced NSCLC patients with driver gene mutations did not achieve an improvement in OS or PFS $(17,23,24)$. Accordingly, the National Comprehensive Cancer Network (NCCN) guidelines recommend against immunotherapy for patients with EGFR gene mutations (25). Regarding the mechanism, many researchers have proposed that $E G F R$ gene mutations can regulate the activation of the PD-1 pathway and reduce the expression of $P D-L 1$ (26); however, others, such as Azuma et al. (27) have asserted the contrary, stating that the expression status of PD-L1 cannot explain the failure of patients with driver gene mutations to benefit from immunotherapy. Another hypothesis is that EGFR gene mutations lead to a low tumor mutation burden (TMB) and reduced immunogenicity. Results of studies of immunotherapy in melanoma (28) have confirmed that only a small number of neoantigens are derived from sensitive gene mutations. For patients with sensitive gene mutations, molecular targeted therapy is still the preferred treatment option.

In clinical trials of advanced NSCLC, patients with positive PD-1/L1 expression have shown superior survival relative to patients with no PD-1/L1 staining (29). However, in the neoadjuvant immunotherapy studies of early NSCLC, PD-1/L1 expression was not found to be clearly correlated with patient's pathological remission benefit. Patients with a higher TMB seem to have better MPR in neoadjuvant immunotherapy (9), but the results of the LCMC 3 trial (30) do not support this conclusion. The mechanism of immunotherapy is different from that of chemotherapy, and there is thus a need for research to explore predictive markers more accurate than PD-1/ L1 and TMB. This way, more accurately personalized treatment plans can be formulated to bolster the benefits of neoadjuvant immunotherapy.

Single-agent or combination therapy with immunotherapy has achieved promising clinical results. Neoadjuvant immunotherapy has several advantages. First, compared with neoadjuvant or adjuvant chemotherapy, which has a grade 3-4 adverse reaction rate of about $40 \%$ (31), neoadjuvant immunotherapy has achieved quite good results. Overall safety, treatment-related adverse reactions above grade 3 are only cases. Second, some studies of neoadjuvant immunotherapy reported achieving MPR at a rate nearly twice of that in studies with chemotherapy (32). For 
example, the 1-year disease-free survival (DFS) rate of stage III patients in the LCMC 3 trial also reached a level very close to that of stage I and II patients (30). Third, intact tumors may have strong host immune characteristics that may be stimulated to release tumor neoantigens, thereby inducing a broader and longer-lasting antitumor effect than that found in the adjuvant setting. Animal studies support this hypothesis, but its specific mechanism of action remains to be explored (20). Evidence from small-scale phase II clinical trials remain insufficiently convincing, and the relevant mechanisms and advantages of neoadjuvant immunotherapy need to be explored in more depth in phase III clinical trials.

\section{Consensus 3: the strategy of neoadjuvant immunotherapy for NSCLC}

* The combination of neoadjuvant immunotherapy and platinum doublet chemotherapy can be more advantageous compared to the adjuvant approach. Neoadjuvant single-agent immunotherapy needs to be further explored in larger studies.

* A total of 2-4 cycles of immunotherapy is recommended, with evaluation after 2 cycles to assess response.

* A dual-immunotherapy neoadjuvant regimen of PD-1/L1 combined with anti-CTLA-4 may be worth considering, but further evidence is needed.

* Other studies are exploring neoadjuvant immunotherapy combined with antiangiogenic drugs or radiotherapy, the results of which are pending.

In the early stage of the exploration of neoadjuvant immunotherapy, a number of clinical trials of single-agent neoadjuvant immunotherapy were carried out. In 2018, Forde et al. (9) reported 21 cases of stage I-IIIA NSCLC patients (NCT02259621) treated with 2 cycles of singleagent nivolumab neoadjuvant immunotherapy. Among them, 20 patients achieved R0 resection with an MPR rate of $45 \%$, with 2 patients (10\%) achieving pathological complete response (pCR). Despite the initial promising results, the MPR rate of other studies using single-agent neoadjuvant immunotherapy was not as encouraging. For example, the MPR rate of nivolumab monotherapy in the NEOSTAR study was only $17 \%$ with a pCR of $9 \%$ (33). Additionally, the interim data of LCMC3, updated at the 2020 World Lung Cancer Conference, showed an MPR and pCR rate of $21 \%$ and $7 \%$, respectively, in 159 patients who met the enrollment criteria and received surgical treatment (30).
In terms of safety, the timing of surgery has not been significantly delayed, and the related adverse reactions are within the tolerable range. In the study by Forde et al. (9), 4 of the 159 surgical patients in the LCMC3 study were delayed of surgery due to treatment-related adverse reactions, and finally all completed the surgical treatment (30).

Most clinical trials related to neoadjuvant immunotherapy in NSCLC are immunotherapy combined with chemotherapy, generally indicating a higher MPR rate compared to singleagent neoadjuvant immunotherapy. For example, a clinical study (NCT02716038) in the treatment of stage IB-IIIA NSCLC with neoadjuvant atezolizumab combined with chemotherapy has achieved MPR and pCR rates of $57 \%$ $(17 / 30)$ and $33 \%(10 / 30)$, respectively (34). The SAKK 16/14 study (NCT02572843) investigated 3 cycles of cisplatin/ docetaxel followed by 2 cycles of durvalumab in the treatment of stage IIIA (N2) NSCLC patients. Patients who received neoadjuvant immunotherapy had an imaging response rate (from $44.8 \%$ to $58.1 \%$ ) after chemotherapy (35). Among the 55 patients who underwent surgical resection, the MPR rate was $60 \%(33 / 55)$ and the pCR rate was $18.2 \%(10 / 55)$. Thirty-seven patients $(67.3 \%)$ observed postoperative lymph node staging decline. The 1-year event-free survival (EFS) rate was $73.3 \%$. The NADIM study explored the effects of preoperative nivolumab combined with paclitaxel and carboplatin chemotherapy in drive mutation negative, stage IIIA (N2/T4N0) NSCLC patients (36): there was a high MPR rate at $83 \%(34 / 41), 63 \%(26 / 41)$ of the patients achieved pCR, the 2-year PFS rate was $77.1 \%$, and the 2 -year OS rate was $89.9 \%$. Although the incidence of side effects of combined and immunotherapy chemotherapy compared with immune monotherapy was higher, it was also within the tolerable range. In the SAKK 16/14 study, the proportion of patients with side effects above grade 3 was $88.1 \%$ (35). Whether the higher incidence of side effects is related to the trial design of 3 courses of chemotherapy followed by sequential 2 courses of immunotherapy still needs to be verified. The rate of grade $\geq 3$ adverse events (AEs) in 2 other clinical trials of concurrent immunotherapy and chemotherapy was $50 \%$ (34) and $46 \%$ (37), and there were no treatment-related deaths. At present, most of the phase III randomized controlled studies use immunotherapy combined with chemotherapy as the experimental group, which will provide important evidence for the neoadjuvant mode of immunotherapy.

At present, there is no clear guideline recommendation for the dose and interval of neoadjuvant immunotherapy 
before surgery, but several drugs have their usual doses (Tables 1,2). The interval between cycles is generally 2 weeks for nivolumab, durvalumab, and 3 weeks for pembrolizumab, atezolizumab, and structure optimized tislelizumab. In terms of medication and treatment course, most studies choose 2 to 4 cycles. This is the result of comprehensive consideration of various factors, such as efficacy, timing of surgery, patient compliance, and economic conditions, but a higher level of clinical evidence is required to determine the optimal medication regimen.

Although the data for nivolumab plus ipilimumab (antiCTLA-4) in advanced NSCLC are encouraging (38), additional clinical trials investigating the combination of dual immunotherapy in the neoadjuvant setting are needed. Reuss et al. (39) reported the first trial of an arm investigating neoadjuvant and adjuvant nivolumab plus ipilimumab (NCT02259621) in patients with resectable stage IB-IIIA, treatment-naïve NSCLC with planned resection. Due to toxicity, the study arm was terminated early by investigator consensus after 9 of 15 patients were enrolled due to $6(67 \%)$ patients experiencing treatmentrelated adverse events (TRAEs) and 3 (33\%) experiencing grade $\geq 3$ TRAEs. Cascone et al. (33) also reported the result from the NEOSTAR study. In 37 patients resected in the trial, nivolumab and nivolumab + ipilimumab produced MPR rates of $24 \%(5 / 21)$ and $50 \%(8 / 16)$, respectively. Compared with nivolumab, nivolumab + ipilimumab resulted in higher pCR rates $(10 \%$ vs. $38 \%)$; less viable tumor (median $50 \%$ vs. 9\%); and greater frequencies of effector, tissue-resident memory, and effector memory $\mathrm{T}$ cells. In this study, there was no significant difference between the single-drug group and the double-drug group in terms of safety. However, in the melanoma study, the grade 3-4 AEs rate of the dual-immune neoadjuvant regimen was $55 \%$, which was much higher than the $16.3 \%$ of the single-agent treatment (40). Therefore, the toxicity caused by the dual-immune neoadjuvant regimen in the treatment of NSCLC still needs further evaluation.

A growing number of neoadjuvant immunotherapy and radiotherapy/chemoradiotherapy trials are also ongoing (MEDI4736, NCT03217071). An interim analysis of neoadjuvant chemoradiation and durvalumab in the potentially resectable phase III NSCLC trial showed that 18 of the 24 eligible patients who received neoadjuvant therapy simultaneously received surgery. The MPR rate was $77.8 \%$ [14/18, 95\% confidence interval (CI): $54.3-91.5 \%$ ], and the pCR rate was $38.9 \%$ (7/18, 95\% CI: 20.2-61.5\%) (41).

Several studies have demonstrated the promising efficacy of PD-1 plus angiogenesis inhibition agents in advanced NSCLC (42); several other studies have attempted this combination in perioperative treatment, such as pembrolizumab plus ramucirumab (NCT04040361), and sintilimab plus bevacizumab and chemotherapy (NCT03872661).

\section{Consensus 4: efficacy evaluation of neoadjuvant immunotherapy for NSCLC}

* The current Response Evaluation Criteria in Solid Tumors (RECIST) standards may not reflect response assessment with immunotherapy.

* Positron emission tomography-computed tomography (PET-CT) is the preferred modality to evaluate the benefit of neoadjuvant immunotherapy.

* Tumor markers, or circulating tumor DNA (ctDNA) load monitoring may be used to assess treatment response.

* After neoadjuvant immunotherapy, an experienced pathologist should evaluate the resected specimen for MPR and pCR.

* Limited prospective data are available for MPR and the pCR for predicting long-term survival.

* OS and DFS can be used as the endpoint of neoadjuvant immunotherapy.

The current RECIST are an important predictor of OS in NSCLC patients (43). However, the histopathological response of $41-45 \%$ of patients may be inconsistent with CT evaluation when neoadjuvant immunotherapy is used $(9,44)$. Changes in fibrotic components may affect the CT findings, resulting in the CT imaging being unable to accurately evaluate the histopathological response after neoadjuvant therapy.

Although it is rare, hyperprogression is also one of the potential issues related to immunotherapy. After immunotherapy, some patients may experience accelerated disease progression with rapid clinical deterioration (45). The molecular mechanism underlying this process is not yet fully understood, and the proportion accounts for about $10 \%$ of the total cases $(46,47)$. Other patients experience "pseudoprogression"; that is, tumor enlargement in imaging, but pathologically, most of them are caused by tumor necrosis rather than tumor cell proliferation. Hyperprogression and pseudoprogression also increase the difficulty of evaluating the effect of immunotherapy. Generally speaking, the standard uptake value (SUV) of PET-CT in pseudoprogressive tumors is low, and a large 


\begin{tabular}{|c|c|c|c|c|c|c|c|c|c|c|c|c|}
\hline$\stackrel{\infty}{\infty}_{\infty}^{\bar{N}}$ & 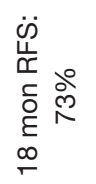 & $\Sigma$ & 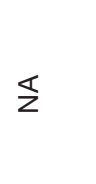 & 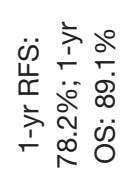 & $\Sigma$ & $\S$ & $\mathbb{z}$ & 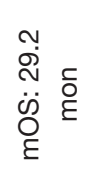 & 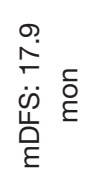 & 定 & 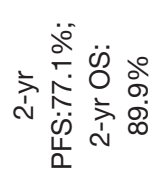 & 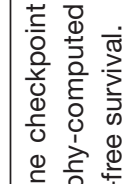 \\
\hline 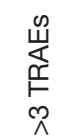 & $\begin{array}{l}\widehat{0} \\
\text { is } \\
-\end{array}$ & $\begin{array}{l}\widehat{o} \\
\stackrel{0}{0} \\
\stackrel{\sigma}{\sigma}\end{array}$ & 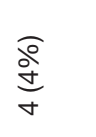 & $\mathbb{z}$ & $\mathbb{z}$ & $\begin{array}{l}\text { F } \\
\text { 吾 } \\
\frac{\mathbb{D}}{0} \\
-\end{array}$ & 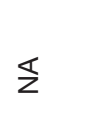 & $\begin{array}{l}\widehat{o} \\
0 \\
\stackrel{+}{ \pm} \\
\mp\end{array}$ & 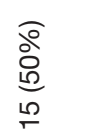 & $\begin{array}{l}\widehat{o} \\
\infty \\
\infty \\
\infty \\
\infty \\
\infty\end{array}$ & 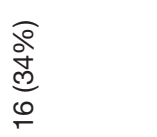 & 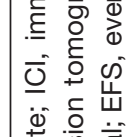 \\
\hline 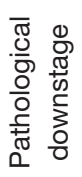 & $\begin{array}{l}\bar{o} \\
\text { oे } \\
\text { d }\end{array}$ & 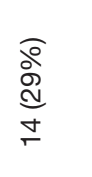 & 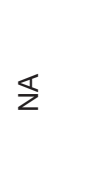 & $\S$ & $\mathbb{z}$ & $\S$ & $\S$ & $\mathbb{z}$ & 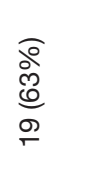 & $\begin{array}{l}\bar{\alpha} \\
\stackrel{0}{\hat{e}} \\
\hat{m}\end{array}$ & 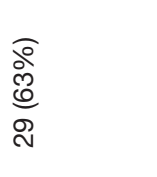 & 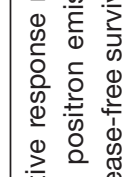 \\
\hline 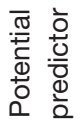 & $\sum_{i}^{\infty}$ & $\begin{array}{l}\frac{5}{1} \\
\frac{1}{4}\end{array}$ & $\S$ & $\S$ & 'َ & 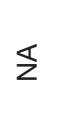 & $\S$ & $\S$ & 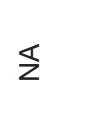 & 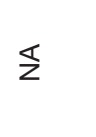 & 立 & 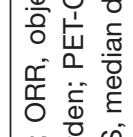 \\
\hline $\begin{array}{l}\stackrel{q}{\widetilde{\alpha}} \\
0\end{array}$ & $\begin{array}{l}\text { ò } \\
\text { Oे } \\
\text { N }\end{array}$ & $\begin{array}{l}\text { वें } \\
\text { @्ञ } \\
\infty\end{array}$ & $\S$ & $\frac{\widehat{o}}{\stackrel{े}{\sigma}}$ & 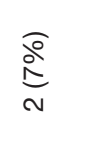 & $\S$ & $\mathbb{z}$ & 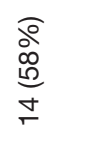 & 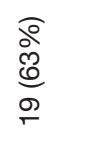 & $\mathbb{z}$ & $\begin{array}{l}\overparen{o} \\
i \\
\stackrel{0}{10} \\
0 \\
m\end{array}$ & $\frac{0}{0}$ \\
\hline $\begin{array}{l}\text { 뜽 } \\
\text { }\end{array}$ & $\underset{\sim}{\stackrel{0}{\circ}}$ & $\begin{array}{l}\overline{8} \\
\stackrel{0}{0} \\
0\end{array}$ & $\begin{array}{l}\widehat{o} \\
b \\
\stackrel{b}{\sigma}\end{array}$ & 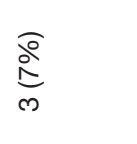 & 0 & $\begin{array}{l}\bar{o} \\
\stackrel{o}{a} \\
\sim\end{array}$ & $\begin{array}{l}\bar{\alpha} \\
\frac{\partial}{\sqrt{a}}\end{array}$ & $\mathbb{z}$ & $\begin{array}{l}\text { ळे } \\
\text { ले } \\
\text { 음 }\end{array}$ & $\begin{array}{l}\text { } \\
\infty \\
\stackrel{0}{\circ} \\
ㅇ\end{array}$ & 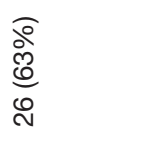 & రั \\
\hline$\frac{\tilde{a}}{\frac{\alpha}{\Sigma}}$ & $\begin{array}{l}\frac{\widehat{o}}{b} \\
\frac{b}{0} \\
\sigma\end{array}$ & 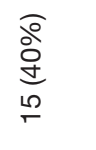 & 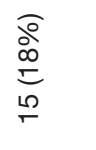 & 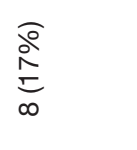 & 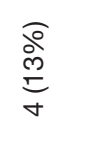 & \begin{tabular}{l}
$\widehat{a}$ \\
$\stackrel{a}{y}$ \\
\multirow{2}{*}{}
\end{tabular} & \begin{tabular}{l} 
बे \\
$\stackrel{\text { \} }{0}} \\
{0}$ & $\mathbb{z}$ & $\begin{array}{l}\frac{a}{\partial} \\
\hat{n} \\
\hat{n}\end{array}$ & $\begin{array}{l}\bar{o} \\
\text { oे } \\
\text { ल) }\end{array}$ & $\begin{array}{l}\widehat{o} \\
\grave{\infty} \\
\infty \\
\text { ले }\end{array}$ & 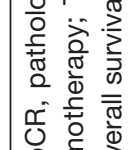 \\
\hline 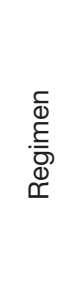 & 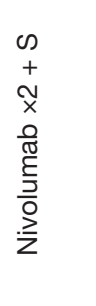 & 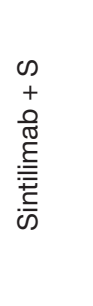 & 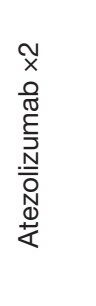 & 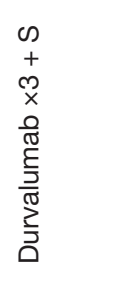 & 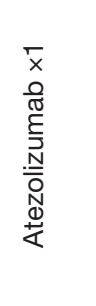 & $\begin{array}{l}\infty \\
+ \\
+ \\
\frac{0}{0} \\
\underline{\Xi} \\
\frac{3}{0} \\
\frac{3}{z}\end{array}$ & 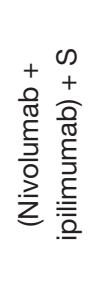 & 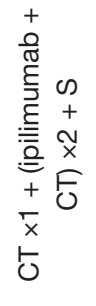 & 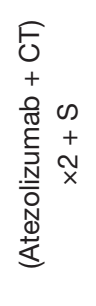 & 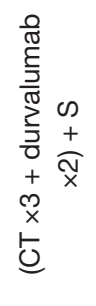 & 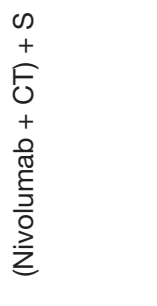 & 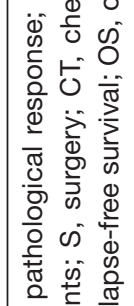 \\
\hline 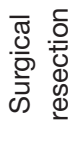 & 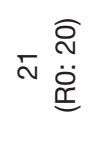 & $\hat{m}$ & ৪৪ & $\underset{\forall}{\forall}$ & 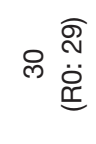 & $\bar{N}$ & $\stackrel{\circ}{\circ}$ & $\stackrel{m}{\sim}$ & 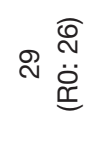 & م & テ & $\begin{array}{l}\tilde{E} \\
\tilde{\sigma}\end{array}$ \\
\hline 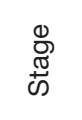 & $\stackrel{\S}{\underline{I}}$ & $\frac{\varrho}{\overline{\underline{1}}}$ & 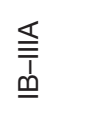 & $\begin{array}{l}\leqq \\
\stackrel{\S}{1} \\
\underline{9}\end{array}$ & $\$$ & $\stackrel{\S}{\underline{I}}$ & & $\begin{array}{l}₫ \\
\stackrel{\Xi}{1} \\
\underline{9}\end{array}$ & 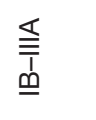 & $\begin{array}{l}\widehat{\mathbb{J}} \\
\leqq\end{array}$ & $\begin{array}{l}\underset{\text { }}{\geqq} \\
\leqq\end{array}$ & 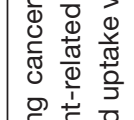 \\
\hline$z$ & $\approx$ & g & 흐 & $\mathscr{q}$ & ৪ి & $\stackrel{\widetilde{N}}{ }$ & $\bar{N}$ & $\stackrel{J}{N}$ & ి్ల & $\stackrel{\infty}{\infty}$ & $\mathscr{q}$ & $\pi$ \\
\hline$\frac{b}{c}$ & 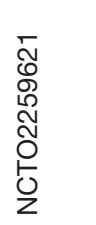 & 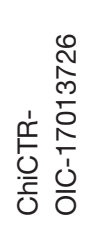 & 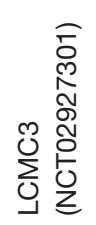 & $\begin{array}{l}\text { O } \\
\mathscr{N} \\
\text { ํ. } \\
\underline{0}\end{array}$ & 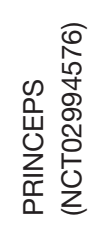 & 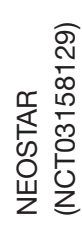 & & 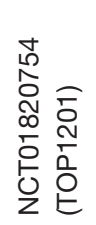 & 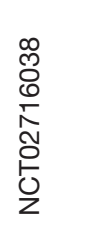 & 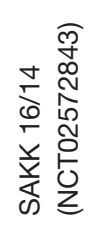 & 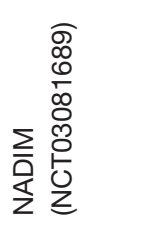 & 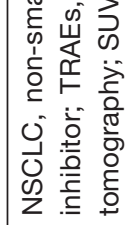 \\
\hline
\end{tabular}
\end{tabular}




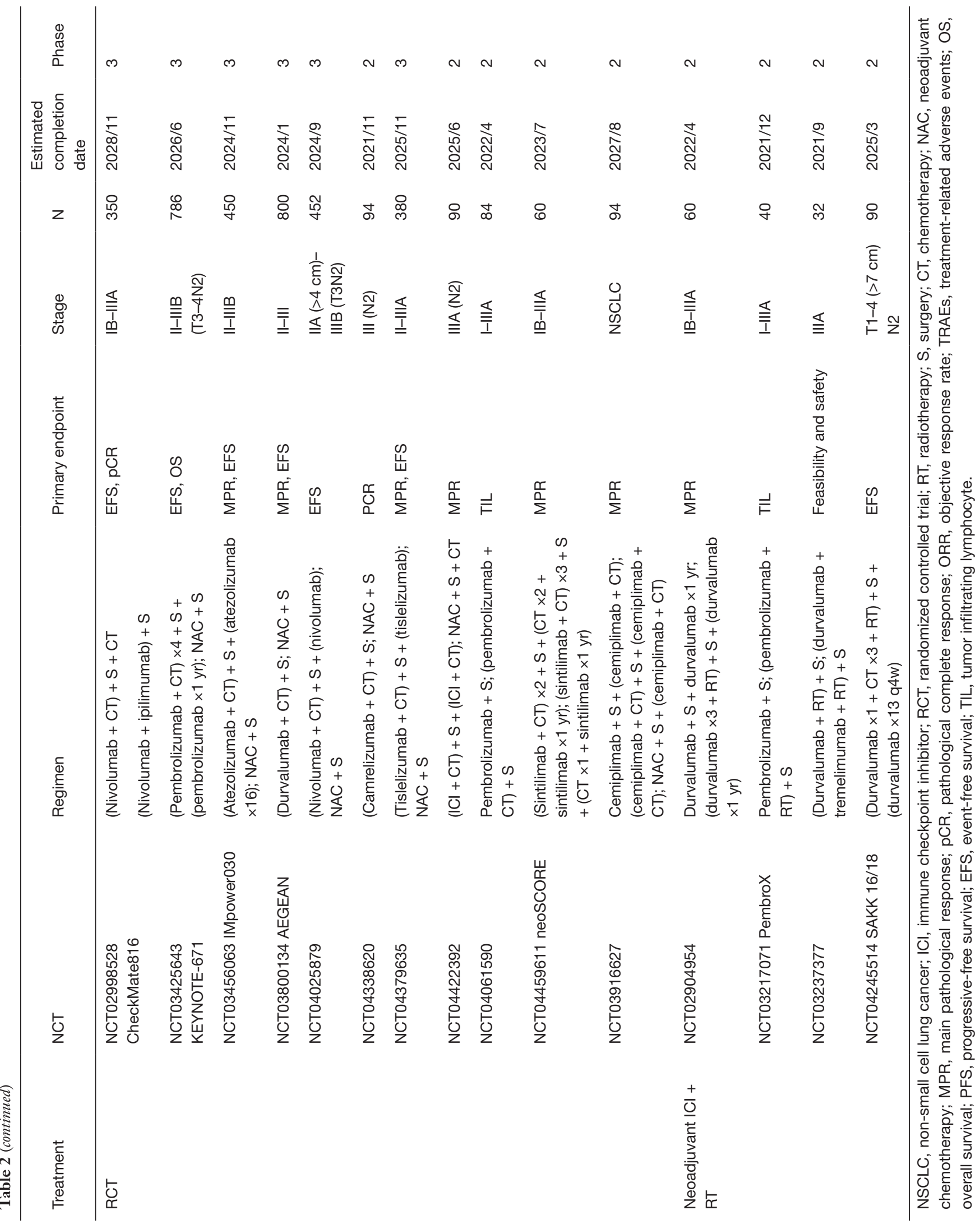


amount of CD8+ T cell infiltration can be seen on the tumor biopsy, which can be used for demarcation (48). Ongoing in-depth study of tumor imaging omics in the field of PET-CT indicates that, compared with CT for lung cancer efficacy monitoring and prognosis judgment, ${ }^{18}$ F-fluoro-2-deoxy-D-glucose $\left({ }^{18} \mathrm{~F}\right.$-FDG) PET-CT imaging omics has higher measurable reliability and stability (49). However, the use of PET-CT to evaluate the efficacy of neoadjuvant treatment for NSCLC is still in its infancy, and there is currently a lack of standardized and prospective data. Further collection and analysis of functional imaging evaluation data before and after treatment is needed to accumulate the relevant clinical experience required for developing the evaluation standards of the future. For example, in one trial (NCT04586465), dynamic PET/CT was used to assess the response of stage IIA-IIIB NSCLC to the neoadjuvant drug pembrolizumab combined with chemotherapy.

Long-term survival data takes about 10 years to collect in early-stage NSCLC. Therefore, MPR has become a possible alternative end point for clinical trials. MPR was first described by Junker et al. (50), who found that some patients with tumor IIB or III regression grades (residual tumor $<10 \%$ ) have significantly improved survival than do other patients. Some subsequent studies have found a significant correlation between MPR and OS (51). In 2014, MPR was more formally recognized as an alternative indicator of OS (52). At present, there is no report detailing the 5 -year survival rate for immunotherapy neoadjuvant studies, but the data on MPR look promising. Nonetheless, pathological remission still has not been validated as a surrogate marker for OS, which remains one of the most relevant end points.

\section{Consensus 5: surgical strategy after neoadjuvant immunotherapy for NSCLC}

* After neoadjuvant immunotherapy with (or without) chemotherapy is administered, minimally invasive surgery can be considered.

* After neoadjuvant immunotherapy is administered, anatomic resection plus mediastinal lymph node sampling ( \pm dissection) is still the standard of care. At this point, there is no evidence showing that neoadjuvant immunotherapy increases surgery-related complications; however, changes in the tumor tissue are expected, and this may impact surgical outcomes.

* Highly difficult operations such as bronchial sleeve lobectomy after neoadjuvant immunotherapy are feasible. * Surgery after neoadjuvant immunotherapy needs to be performed by an experienced thoracic surgeon.

The feasibility and safety of lung resection after neoadjuvant immunotherapy warrant further study. Neoadjuvant immunotherapy may cause mediastinal and hilar fibrosis (53). Bott et al. (54) pointed out that although it is technically challenging, pneumonectomy is feasible and does not increase morbidity or mortality. Similarly, Yang et al. (37) confirmed the feasibility and safety of lung resection after neoadjuvant ipilimumab combined with chemotherapy, while another study suggested a change from lobectomy to thoracotomy is possible during surgery (55). Overall, neoadjuvant chemotherapy and minimally invasive lobectomy can be used for stage IIIA (N2) NSCLC (56), but it has been found that only $25.7 \%$ of patients undergo lobectomy (57).

Neoadjuvant immunotherapy and chemoimmunotherapy may alter the primary tumor vascularization and microenvironment, resulting in adhesions and fibrosis, and increasing the difficulty and duration of surgery. At present, there is no evidence that the rate of conversion from minimally invasive resection to open thoracotomy increases as the complication rate increases. Despite this, thoracotomy does not seem to significantly affect morbidity or early mortality (54). After lung resection, the pathologist can determine the pathological response, the predictive factors of MPR, and the potential impact on survival.

In addition, neoadjuvant immunotherapy has been shown to not significantly reduce the completion of surgery. In the NADIM study, $89 \%(41 / 46)$ of the patients completed the operation, all with R0 resection, and $93 \%(38 / 41)$ of the patients reached clinical downgrade (36). However, from the experience from several case series, many patients have thoracic adhesions. Some patients have severe adhesions in the lymph nodes and surrounding tissues after neoadjuvant treatment, making clean-up difficult to, while ICI-related diseases, such as immune pneumonia after surgery, may also occur.

Liang et al. (58) reported the first sleeve lobectomy cohort after neoadjuvant immunotherapy plus chemotherapy, concluding that sleeve lobectomy for advanced NSCLC following chemoimmunotherapy is feasible. Although the operation was more complex, neoadjuvant chemoimmunotherapy did not delay postoperative recovery. The study also indicated that greater destruction on the elastic fiber of the blood vessels, vascular wall degeneration, fibrinoid necrosis and fibrosis, 
and greater pulmonary interstitial exudation were found in neoadjuvant chemoimmunotherapy patients compared to the neoadjuvant chemotherapy patients.

\section{Consensus 6: management of AEs during neoadjuvant immunotherapy for NSCLC}

* Perioperative immunotherapy of special populations (patients with autoimmune diseases, patients after organ transplantation, patients with chronic viral infections, patients with immunodeficiency, pulmonary fibrosis, pregnancy, liver or kidney dysfunction, etc.) requires extra caution.

* Neoadjuvant immunotherapy for patients with operable NSCLC is relatively safe, and the incidence of all grades and $\geq 3$ grades of AEs with single-agent immunotherapy is $23-57 \%$ and $4.5-13 \%$, respectively.

* During perioperative immunotherapy, it is necessary to regularly check and monitor organ functions (laboratory tests and imaging examinations, lung function, electrocardiogram, thyroid function and other baseline evaluations). Early identification of severe immunerelated pneumonia and immune-related cardiotoxicity is important.

* The treatment of AEs of perioperative NSCLC immunotherapy should be performed according to the treatment principles and countermeasures of advanced stage of NSCLC.

* Patients that suffered from severe immune-related adverse events (irAEs) who meet the referral indications should be transferred/referral (a medical institution with a qualified irAEs MDT team) as soon as possible.

As the clinical benefit of neoadjuvant immunotherapy is being lauded, the increase of immunotherapy-related toxic and adverse effects (AEs) should not be ignored. In a few cases, these events may be life-threatening, resulting in inoperability, delayed surgery, and increased postoperative complications (59). A meta-analysis conducted by the Lung Adjuvant Cisplatin Evaluation (LACE) and NSCLC Collaborative Group $(6,59)$ showed that neoadjuvant therapy reduces the risk of death by $13 \%$, and adjuvant chemotherapy improves the 5 -year survival rate of patients by $5.3 \%$ [hazard ratio $(\mathrm{HR})=0.89$ ]; however, the incidence of grade 3-4 AEs is as high as $66 \%$. Although the incidence of most grade 3-4 AEs in neoadjuvant immunotherapy compared with chemotherapy alone is not significantly higher (60), the associated irAEs, especially immune-related pneumonia, cardiotoxicity, gastrointestinal toxicity, and other rare but severe-toxicity events can seriously worsen the prognosis of patients (61). Therefore, there is an urgent need for more refined management of irAEs in clinical practice for neoadjuvant immunotherapy.

Neoadjuvant immunotherapy is usually completed in 2-4 cycles. Some small-sample phase II clinical studies focused on exploring the impact of immunotherapy on surgical procedures. The LCMC3 study initially reported 101 patients with early resectable NSCLC (30). After 2 cycles of atezolizumab before surgery, the incidence of grade 3-4 AEs was $29 \%$ and mainly included fatigue, fever, loss of appetite, elevated transaminase, nausea, joint pain, flu-like symptoms, diarrhea, pneumonia, anemia, etc.; however, these were generally well tolerated by patients, and there was no delay in surgery.

The NEOSTAR study evaluated nivolumab vs. nivolumab combined with ipilimumab dual-agent neoadjuvant therapy (33). There was no significant difference in the incidence of AEs between the two groups. The incidence of grade 3-5 AEs was as follows: hypermagnesemia 4\%, low oxyemia $4 \%$, severe diarrhea $4 \%$, and hyponatremia $4 \%$. Among the patients, 1 in the single-drug group did not undergo surgery due to serious AEs, while 4 in the dualdrug group did not receive surgery.

A study evaluating the safety of 20 patients with nivolumab single-agent neoadjuvant immunotherapy for resectable NSCLC (stage IA-IIIA) reported 13 patients expecting to receive minimally invasive treatment (thoracoscopy) before surgery (54). After neoadjuvant immunotherapy, 7 patients $(53.8 \%)$ eventually converted to thoracotomy due to hilar inflammation or fibrosis. Among them, the conversion rate of stage IA patients was $25 \%(1 / 4)$, and the conversion rate of stage IB-IIIA patients was $67 \%$ $(6 / 9)$. The incidence of postoperative atrial arrhythmia was about $30 \%(6 / 20)$, myocardial infarction was $5 \%(1 / 20)$, lung infection was $5 \%(1 / 20)$, pulmonary embolism was $5 \%$ $(1 / 20)$, and empyema was $5 \%(1 / 20)$.

The NEOSTAR study examined the preoperative single-agent nivolumab treatment for 2 cycles and reported the following incidences of postoperative complications: persistent lung leak $22 \%$, bronchopleural fistula $9 \%$, empyema $4 \%$, lung infection $4 \%$, and nonspecific pneumonia $4 \%$ (33). The latest report of the NADIM study showed that after 3 cycles of preoperative nivolumab combined with carboplatin and paclitaxel chemotherapy, the postoperative complications rate was $17.1 \%(7 / 41)$ and included arrhythmia, persistent lung leakage, and respiratory tract infection, postoperative pain, recurrent 
Table 3 Postoperative adjuvant immunotherapy for NSCLC

\begin{tabular}{|c|c|c|c|c|}
\hline Trial & Eligible patients & Intervention following surgery & $\begin{array}{l}\text { Estimated } \\
\text { enrolment }\end{array}$ & $\begin{array}{l}\text { Primary } \\
\text { endpoint }\end{array}$ \\
\hline IMpower010 & Stage IB-IIIA NSCLC & $\begin{array}{l}\text { Arm A: platinum doublet ( } 4 \text { cycles) then atezo (16 } \\
\text { cycles); arm B: platinum doublet ( } 4 \text { cycles) then } \\
\text { best supportive care }\end{array}$ & $\mathrm{N}=1,280$ & DFS \\
\hline ANVIL & Stage IB-IIIA NSCLC & $\begin{array}{l}\text { Arm A: (optional chemotherapy and RT) } \\
\text { nivolumab (1 year); arm B: (optional chemotherapy } \\
\text { and RT) observation }\end{array}$ & $\mathrm{N}=903$ & DFS, OS \\
\hline PEARLS/KEYNOTE-091 & Stage IB/II-IIIA NSCLC & $\begin{array}{l}\text { Arm A: (optional chemotherapy) pembro (1 year); } \\
\text { arm B: (optional chemotherapy) placbo ( } 1 \text { year) }\end{array}$ & $N=1,080$ & DFS \\
\hline BR31 & Stage IB-IIIA NSCLC & $\begin{array}{l}\text { Arm A: (optional chemotherapy and RT if N2) } \\
\text { durva (1 year); arm B: (optional chemotherapy } \\
\text { and RT if N2) placebo (1 year) }\end{array}$ & $\mathrm{N}=1,360$ & DFS \\
\hline
\end{tabular}

NSCLC, non-small cell lung cancer; Chemo, chemotherapy; IO, immunotherapy; RT, radiotherapy; OS, overall survival; DFS, disease-free survival.

laryngeal nerve palsy, thrombocytopenia, postoperative lung infection, lower limb cellulitis, atrial fibrillation (36).

In general, neoadjuvant immunotherapy for patients with operable NSCLC is relatively safe, and the incidence of all grades and $\geq 3$ grades of AEs with single-agent immunotherapy is $23-57 \%$ and $4.5-13 \%$, respectively. However, current neoadjuvant immunotherapy research consist almost completely of phase I/II small-sample exploratory studies, with short follow-up times and incomplete data. It is will be impossible to obtain the full picture concerning neoadjuvant irAEs until more large-scale and prospective studies are completed. Good and standardized perioperative immune adverse reaction management can not only ensure the smooth implementation of the overall treatment plan but also has a positive influence in improving the clinical outcome of patients.

\section{Consensus 7: adjuvant immunotherapy after surgery for NSCLC}

* The postoperative adjuvant treatment plan for NSCLC should be carried out in accordance with the NCCN guidelines.

* After postoperative adjuvant chemotherapy, maintaining immunotherapy for up to 1 year should be considered.
* Detection of minimal residual disease (MRD) shortly after surgery and changes in ctDNA during adjuvant treatment may predict prognosis, but further data are needed before this strategy to be can be used in clinical practice.

Several studies were designed to explored the efficacy of adjuvant immunotherapy after surgery for NSCLC (IMpower010, ANVIL, PEARLS, BR31, ALCHEMIST; Table 3). IMpower010 is a global, multicenter, open-label, randomized trial (NCT02486718), comparing the safety and efficacy of adding atezolizumab to platinum doublet with best support care in an adjuvant setting of NSCLC patients. An early report from the IMpower010 study showed that the study has met the primary end point of improvement in DFS with atezolizumab versus use of best supportive care as treatment for patients with completely resected stage II/IIIA NSCLC. At the time of the report, atezolizumab did not significantly affect DFS in the intention-to-treat analysis for all stage IB-IIIA NSCLCs that were randomized into the study. Longer follow-up, including of OS, will be needed to fully assess the efficacy results of this study.

ctDNA may be an effective tool for noninvasive monitoring of treatment response and has high specificity and sensitivity for predicting disease recurrence. It has great potential for cancer-specific molecular changes and has broad application prospects. Researchers believe that 
performing individualized deep sequencing of ctDNA on tumors can diagnose molecular residual tumors early and facilitate personalized adjuvant therapy as early as possible when the tumor burden is lowest. Because NSCLC is still undergoing gene mutations during the treatment process and the half-life of ctDNA is very short, real-time monitoring is required to determine the efficacy and resistance of the drug. Therefore, future clinical trial design should incorporate study of clinical treatment efficiency, continuous collection, verification of liquid biopsy specimens, and MPR and pCR to identify the benefit population of neoadjuvant or adjuvant therapy.

\section{Discussion}

Compared with chemotherapy and targeted therapy, immunotherapy has unique advantages in its therapeutic principles. In theory, stimulating the activity of the body's immune cells can better mitigate tumor resistance. Judging from the MPR rate of single-agent and combination chemotherapy, the prospect of immunotherapy application in lung cancer is very optimistic. In the next 5-10 years, large-scale phase III randomized controlled trials and evaluation of long-term survival will be the focus of immunotherapy research.

However, some issues related to neoadjuvant immunotherapy still need to be resolved. First, reports suggest that some patients cannot receive follow-up treatment due to disease progression during neoadjuvant treatment. Surgeons need to be cautious in considering the potential risks of neoadjuvant treatment before surgery. Second, the preoperative medication cycle of the current clinical research of neoadjuvant immunotherapy is inconsistent, ranging from 2 to 4 cycles. Recently, Liu et al. (62) found through mouse animal models that preoperative immunotherapy can affect the survival of tumor-negative mice. Preoperative immunotherapy that is too long or too short can reduce the efficacy. The above study suggests that for preoperative neoadjuvant treatment, it is still necessary to explore the best preoperative treatment time. Third, in the neoadjuvant immunotherapy, the pCR of different immunotherapy drugs and treatment modes are quite diverse. Based on the current clinical research data, the efficacy of PD-1 inhibitors is better than that of PD-L1 inhibitors, and the efficacy of PD-1 inhibitors combined with chemotherapy is better than that of PD-1 monotherapy. More clinical research data are required to verify these observations. Fourth, all current clinical studies of neoadjuvant immunotherapy have no biomarkers to screen NSCLC patients. In the future, the inclusion of markers in the screening criteria may further improve the efficacy of neoadjuvant immunotherapy. Fifth, surgical-related risks and difficulties (such as surgical delay and disease progression) and perioperative complications caused by neoadjuvant targeting and immunotherapy are concerning for surgeons. In addition, how to further "precisely" select suitable patients and explore a more efficient and low-toxicity combination therapy model remains to clarified by future NSCLC neoadjuvant therapy research.

Perioperative immunotherapy provides hope for the long-term survival of NSCLC patients. Future research can help identify an ideal biomarker to reliably predict benefit of immunotherapy, the number of cycles and duration of immunotherapy in the pre- or postoperative setting, and the optimal combination in the perioperative setting.

\section{Questions to be further discussed and considered}

(I) Will you use the neoadjuvant immunotherapy for resectable NSCLC patients?

Federico Cappuzzo: Only in clinical trials.

Junji Uchino: Although more evidence needs to be accumulated, I consider it as a promising treatment. Expanded indications in different countries are required to implement treatment in practice.

Kye Young Lee: Yes, I will. But case selection is necessary. mEGFR-, ALK-, and ROS1-positive cases should be ruled out. In a similar respect, smoking status should be considered.

Mariano Provencio: I am convinced that chemoimmunotherapy is better than chemotherapy alone, and therefore, I am sure I will use it as soon as it is approved by the regulatory authorities

Paul Hofman: Yes. Neoadjuvant immunotherapy is certainly associated with a decrease in tumor TNM stage before surgery, with an increased $\mathrm{R} 0$ resection rate, with preoperative control of possible micrometastases and/or the number of circulating tumor cells (since even early tumors can be associated with a shedding of tumor cells in the blood stream), and, with an improvement of DFS and, more importantly, of the OS of early-stage NSCLC patients (to be confirmed).

Takeo Nakada: Yes, I will. Of the resectable lung cancer 
patients, I consider stage IB-IIIA to be an indication for neoadjuvant immunotherapy. In the future, stage IA3 puresolid NSCLC should be considered as an indication for preoperative chemotherapy.

Toyoaki Hida: Yes.

William Chi-Shing Cho: For patients with resectable lung cancer, if surgical resection is difficult, I will consider giving immunotherapy, or immunotherapy plus chemotherapy before surgery, and then conduct a preoperative evaluation again after two courses of treatment. This is because ICIs, such as PD-1 and CTLA4 , can enhance antitumor immunity by activating antigenspecific $\mathrm{T}$ cells in the tumor. This induces an antitumor $\mathrm{T}$ cell immune response, which can prevent tumor recurrence. In addition, the increase in activated $\mathrm{T}$ cells after neoadjuvant immunotherapy may reduce the severity of immunosuppression after surgery and reduce the possibility of disease recurrence. However, different immunotherapies have different toxicity characteristics involving multiple organs and irAEs; thus, hyperprogession may delay surgery and/or increase the risk of intraoperative complications. The extensive delay in surgical resection due to AEs may lead to tumor progression and poorer outcomes. It may be that neoadjuvant therapy destroys the primary tumor vascularization and microenvironment, leading to adhesions and fibrosis, increasing the difficulty and duration of surgery. In addition, physical fatigue caused by neoadjuvant immunotherapy may extend the recovery time from surgery.

All in all, neoadjuvant immunotherapy is not yet a standard treatment because trials are still in progress, but it may be carried out in the next year or two. It is unclear whether this will be a single-agent checkpoint inhibitor or a checkpoint inhibitor combined with chemotherapy, but my guess is it will be the latter.

Haruhiko Sugimura: Currently, our insurance (government) does not cover it, but we will definitely use it in the near future.

Muhammad Furqan: Yes.

Satoshi Watanabe: I will use neoadjuvant immunotherapy if the survival benefit of the treatment is proven by a phase III trial.

Stefano Bongiolatti: Immunotherapy with PD-1/PD-L1 inhibitors is not the standard of care in my country in the neoadjuvant setting, and ICIs can be used only in clinical trials.

Yusuke Tomita: We need more clinical evidence to use neoadjuvant immunotherapy in clinical practice.

Filippo Longo: We usually do not perform neoadjuvant immunotherapy for resectable NSCLC. For stage IIIAIIIB resectable NSCLC we usually perform induction chemotherapy before radiotherapy.

In-Jae Oh: Yes, but I will use the neoadjuvant I-O within the clinical trials. The South Korean government mostly covers the cost of the global standard of anticancer treatment in NSCLC patients. But our government and many Korean investigators are thinking that the time for neoadjuvant I-O has not yet arrived. I hope to change the Korean standard by adding valuable data from clinical trials.

Nobuhiko Seki: Yes, I will. However, I do expect neoadjuvant immunotherapy for resectable NSCLC patients with negative driver mutations. Furthermore, I think the indications for PD-L1-negative patients will remain controversial until we see the results of DFS and OS of the phase III trial for preoperative neoadjuvant immunotherapy.

Pierfilippo Crucitti: We usually do not perform neoadjuvant immunotherapy for resectable NSCLG. For stage IIIA resectable NSCLC, we usually perform induction chemoradiotherapy before surgery.

Sai Yendamuri: Yes, I would use it for stage IIIA patients. For lower than stage IIIA, I would use it in the context of a clinical trial.

Sang-Won Um: Yes, I will.

Shinji Sasada: I agree with the preoperative use. The reason is that pCR may be obtained.

Ben G. L. Vanneste: My advice would be to use it only in clinical trials.

Dirk De Ruysscher: We are using it now only in clinical studies.

\section{(II) What is the main concern when you use immunotherapy in the neoadjuvant setting?}

Federico Cappuzzo: No concern. I think this is a very effective strategy.

Junji Uchino: There are concerns about cases of hyperprogression and cases of SAE that make surgery difficult.

Kye Young Lee: [My concern is] hyperprogression, which does not happen that infrequently, especially in single immunotherapy. For this reason, I think that neoadjuvant chemoimmunotherapy is better than single immunotherapy except for high PD-L1 expression cases, with 2 cycles of neoadjuvant chemoimmunotherapy being enough.

Mariano Provencio: I would like to have objective markers that could differentiate those patients who will respond from those who will not. I am also concerned about 
the poor relationship between radiological and PET/CT response and pathological response that exists.

Paul Hofman: We need to keep in mind at least three points:

(I) It is mandatory to look for some targetable genomic alterations before administration of neoadjuvant immunotherapy since the latter can be less effective and even more highly toxic in patients having genomic alterations (currently with a strong focus on $E G F R$ and $A L K$, but perhaps soon on other genes such as KRAS, ROS1, RET, MET, $H E R 2$...). So, NGS should be done simultaneously with PD-L1 immunohistochemistry (IHC) and NGS from biopsies before starting any neoadjuvant immunotherapy. Alternative therapy can be for example an adjuvant therapy targeting detected EGFR mutations (del19 and L858). Moreover, bronchial biopsy (and cytological samples such as those obtained during EBUS) may have a few number of tumor cells, making the assessment of PD-L1 expression in these samples difficult.

(II) Currently it is not so well known if neoadjuvant immunotherapy can effectively increase the longterm life of surgically resected NSCLC patients.

(III) Discussion may be arranged between surgeons concerning the impact of neoadjuvant immunotherapy on the feasibility of surgery (since delaying surgery can have an impact on tumor risk progression and on an overtreatment since some surgical complications can sometimes occur after immunotherapy).

Takeo Nakada: The main concern is grade 3-4 AEs that can delay surgical treatment.

Toyoaki Hida: [My concern is] hyperprogressive disease in neoadjuvant immunotherapy without chemotherapy.

William Chi-Shing Cho: The main concern is the safety of this treatment. The optimal timing of surgery after immunotherapy is still unknown. Surgeons are more concerned about the inflammatory consequences of immunotherapy, leading to more hilar fibrosis, hilar scarring, lymph node adhesion, etc. This will lead to more difficult dissections, longer operation time, a higher conversion rate of minimal access technology, and a higher complication rate.

Regarding AEs, various organs may be involved, such as pneumonitis myocarditis, colitis, pituitary inflammation, rash, pneumonia, neuromuscular toxicity, hypothyroidism, and joint pain. There is also a concern with delaying surgery, progression, or hyperprogression of treatment, which can impair the healing process.

The timing of surgery is of concern because shortening the duration of neoadjuvant therapy can increase immune activity and reduce the incidence of irAEs.

I think we can perform biomarker testing, for example, NGS of each patient before starting treatment, because it is important to exclude mutations such as EGFR or $A L K$ before starting chemoimmunotherapy. Of course, efficacy is also an important indicator of concern. According to my experience, neoadjuvant immunotherapy plus chemotherapy has achieved satisfactory results in many patients. The effectiveness of neoadjuvant treatment should be evaluated over time by PET/CT to determine unsuspected metastases and comorbidities.

Haruhiko Sugimura: There are several issues: how do we focus in on the population? (PD-L1 expression? Which stages?) What combination should we choose? (Monotherapy or several combinations with ordinary cytotoxic cancer drugs and several kinds of ICIs?). We have to be cautious so that the timing of the operation is not late and be aware of irAEs.

Muhammad Furqan: The biggest concern is not the immunotherapy. The issue is using a neoadjuvant approach $v s$. an adjuvant one. As medical oncologists, we prefer the neoadjuvant approach, as patients tolerate this therapy better and this can help in eliminating micrometastases. However, surgical colleagues worry about the impedance to surgical feasibility, as a small percentage of patient's may progress during or right after neoadjuvant therapy and will not undergo potentially curable surgery, or patient enthusiasm may dampen from toxicity of the neoadjuvant approach. I think it requires change of culture and will happen over time.

Satoshi Watanabe: There is a risk that patients will not be able to receive radical surgery because of tumor progression during neoadjuvant immunotherapy and irAEs.

Stefano Bongiolatti: In my opinion, the major concern of immunotherapy in the neoadjuvant setting is the lack of large data on long-term oncological outcomes. The reported results on the deep impact of ICIs on MPR and should be associated with improved long-term results, but data on DFS and OS are still weak.

Yusuke Tomita: Although clinical studies report that the timing of surgery has not been significantly delayed, there is a potential risk of treatment delay of surgery in neoadjuvant therapy.

Filippo Longo: We do not use immunotherapy in the 
neoadjuvant setting.

In-Jae Oh: I worried about tumor progression after neoadjuvant I-O resulting in unresectable stage NSCLC. So, we have to find the predictive biomarkers for neoadjuvant I-O with minimal tumor progression.

Nobuhiko Seki: My main concern is that treatmentrelated AEs, hyperprogression, and pseudoprogression may prevent subsequent curative surgery. Alternatively, TRAEs such as pituitary hormone deficiency may force some patients to continue hormone replacement therapy after surgery. Perhaps some of these patients could have been cured by surgery alone.

Pierfilippo Crucitti: We do not use immunotherapy in the neoadjuvant setting.

Sai Yendamuri: The possibility of pneumonitis in a marginal patient may make the patient unresectable in terms of compromised lung function.

Sang-Won Um: Hyperprogression of disease and ICIrelated disease such as pneumonitis are the two main concerns.

Shinji Sasada: [My concern is] surgery being discontinued due to the complication of strong irAEs.

Ben G. L. Vanneste: [My concern is] exacerbation of side effects, difficulties in wound healing, and more complications.

Dirk De Ruysscher: More surgical complications, i.e., more open procedures because of fibrotic tissue in the hilar region of the lung.

\section{(III) How do you choose the adjuvant therapy after the neoadjuvant immunotherapy surgery?}

Federico Cappuzzo: We are not using immunotherapy neoadjuvant outside clinical trials.

Junji Uchino: The evidence is lacking at present, and it is considered that it will not be carried out. Future clinical studies are desirable.

Kye Young Lee: It depends on the final pathologic staging and pathologic response. I do not think that adjuvant therapy is necessary in the case of p-stage IA or IB. In the cases of p-stage II or III with MPR or pCR, adjuvant therapy could be omitted. But in the cases without MPR or pCR, I recommend adjuvant anti-PD-1 single immunotherapy.

Mariano Provencio: I think this is an added problem. Outside of the trial, I think we should stick to the current standard and not use radiotherapy if the resection is complete, and, since several studies include adjuvant immunotherapy postoperatively, maybe it would make sense. I think it is a difficult issue to be very conclusive, yet perhaps liquid biopsy and ctDNA data will clarify who to treat.

Paul Hofman:

(I) Adjuvant therapy depends on the resection rate after neoadjuvant immunotherapy.

(II) It can be of strong interest to follow the patients not only by CT but also using blood biomarkers (such as the quantity/level of ctDNA).

(III) If some potential genomic alterations (such as $E G F R$ mutations) were missed on the preoperative samples (biopsies) and are present in surgical resected specimen (may be due to the tumor heterogeneity and/or the low quantity/quality of DNA extracted from biopsies), it could be of interest to switch to targeted therapy, such as osimertinib after surgery.

(IV) Currently the study of adjuvant immunotherapy for lung cancer is still certainly in the exploratory step with no mature results.

(V) If the resection is not complete, the choice of chemotherapy or chemoradiotherapy can be challenging according to the clinical situation.

(VI) Attention should be given to the MPR, since a low MPR can be associated with early recurrence.

(VII) We can discuss maintaining immunotherapy after surgery in case of weak major pathologic response.

Takeo Nakada: The first choice is continuing to maintain immunotherapy for up to 1 year.

Toyoaki Hida: Adjuvant immunotherapy is administered when ctDNA is detected after surgery, and adjuvant immunotherapy is not administered when ctDNA is not detected after surgery.

William Chi-Shing Cho: This is an unresolved issue for which no consensus has been reached. If the patient has 3 or more cycles of chemotherapy before surgery, I do not recommend doing more in the adjuvant phase. I think the answer will follow the upcoming trials on the correlation between molecular residual disease and the pertinence of neoadjuvant immunotherapy in these subgroups.

Haruhiko Sugimura: Actually, we do not yet have our own experience in this situation.

Muhammad Furqan: [This] needs to be driven by the data. Obviously. If the patient has received neoadjuvant chemotherapy, then no further chemotherapy in the adjuvant setting is needed, but the use of immunotherapy for 6 months to 1 year after the surgery, as is being done 
in the CheckMate 816 and other ongoing studies, may be reasonable.

Satoshi Watanabe: I will choose the adjuvant therapy based on the evidence. For example, adjuvant nivolumab was administered after neoadjuvant chemoimmunotherapy followed by surgery in the CheckMate 77T study. If this study demonstrates a survival benefit, I will use nivolumab as the adjuvant therapy.

Stefano Bongiolatti: Theoretically, we should complete 6 cycles of perioperative therapy regardless of the pathological stage with the same preoperative scheme including ICIs. Adjuvant immunotherapy is recommend for 1 year after surgery only within clinical trials; besides, the evidence in this setting is weak.

Yusuke Tomita: There is no evidence to answer this question. We need to wait for the accumulation of more evidence.

Filippo Longo: For radiochemotherapy-treated patients in a neoadjuvant setting, we usually do not perform adjuvant therapy after surgery. Immunotherapy may be evaluated in case of tumor relapse.

In-Jae Oh: I usually choose the similar regimen which was successfully used as neoadjuvant therapy. In case of adjuvant therapy other than I-O, I want to select platinum doublet chemotherapy regimen such as paclitaxel/cisplatin, vinorelbine/cisplatin, or pemetrexed/cisplatin. They are the standard Korean adjuvant chemotherapeutic agents.

Nobuhiko Seki: Ideally, I would like to provide the adjuvant therapy only to the patients who are concerned about the presence of MRD based on the postoperative ctDNA status. However, I think it will take some time before the measurements of ctDNA are put into practical use. Therefore, I currently believe that adjuvant therapy should be given as much as possible to all patients who may have distant micrometastasis, even to the patients whose pCR has been obtained by the neoadjuvant immunotherapy. By the way, it should be noted that the indications of the adjuvant immunotherapy for the patients showing negative PD-L1 status or positive driver mutations are controversial.

Pierfilippo Crucitti: For radiochemotherapy-treated patients, we usually do not perform adjuvant therapy. Immunotherapy may be evaluated in the case of tumor relapse.

Sai Yendamuri: This depends on the extent of response. If there is complete response, the value of additional therapy is questionable.

Shinji Sasada: If the resected specimen has a good pathological response, the same drug as preoperative treatment will be used. If it does not work, I choose the cytotoxic chemotherapy recommended in the guidelines.

Ben G. L. Vanneste: [This depends] on stage: IA, no; IB is controversial, and one or more high-risk features could be determined, including lymphovascular invasion, poor differentiation, or high SUV on PET, which is variably defined as SUV 10 or higher. Stage II and IIIA patients are candidates.

Dirk De Ruysscher: For stage IC-IIIA, we use 4 cycles of platinum-doublet therapy.

\section{(IV) Which regimen or combination do you prefer to use as the neoadjuvant immunotherapy? Why?}

Federico Cappuzzo: Not applicable.

Junji Uchino: Given the findings of the present clinical studies, nivolumab plus chemotherapy (in the NADIM study) may be the most promising regimen.

Kye Young Lee: I prefer KEYTRUDA monotherapy for 2 cycles in the case of high PD-L1 expression (>50\%). If the tumor PD-L1 expression is low, chemoimmunotherapy maybe better with the consideration of age factor and performance status. Chemotherapy regimen is platinumbased doublet depending on the histology. (paclitaxelbased in squamous cell type and pemetrexed-based in nonsquamous cell type). I think that 2 cycles will be enough for neoadjuvant immunotherapy because cytotoxic T-cells recruitment develops relatively early after immunotherapy.

Mariano Provencio: I have a lot of very good experience with nivolumab and CarboTaxol which is useful in all histologies and has moderate adverse effects.

Paul Hofman: The IONESCO [trial] can be of interest too (for stage IB-II) since the primary end point is also R0 resection. However, in our institution (University Côte d'Azur, Nice, France) we use the ongoing AEGEAN clinical trial (durvalumab + chemo versus chemo + placebo). The MPR seems higher than in some other neoadjuvant immunotherapy options. Nivolumab vs. nivolumab + ipilimumab was of interest (NEOSTAR study) at the beginning since it was for stage I-IIIA (all early stages). However, the MPR was $24 \%$ for primary outcomes and so certainly too weak.

Takeo Nakada: I experienced some clinical trials of neoadjuvant immunotherapy (durvalumab, atezolizumab, nivolumab, and pembrolizumab) plus chemotherapy. However, the number was not enough. Therefore, I cannot answer this question.

Toyoaki Hida: [I prefer] PD-1 inhibitor combined 
with chemotherapy (cisplatin + pemetrexed for NSQ, carboplatin + paclitaxel for SQ) with reference to the results of the CheckMate 816 study.

William Chi-Shing Cho: We need trials to determine this. It seems that most regimens involve platinum-based chemotherapy plus anti-PD-1/PD-L1 drugs. I have seen some good responses to this approach. For adenocarcinoma, I usually select pembrolizumab (KEYTRUDA)/nivolumab (Opdivo) + pemetrexed + platinum, while for squamous cell carcinoma, I select pembrolizumab (KEYTRUDA) + paclitaxel-albumin/gemcitabine + platinum. However, I do not think it is necessary to give cisplatin. We have seen some good responses to the carboplatinum-based regimen. To better evaluate this, we need the best response assessment and biomarker exploration of neoadjuvant immunotherapy.

Haruhiko Sugimura: Nivolmab + ipilimumab may be only one choice as far as the data available to us, or cisplatin, ALIMTA (pemetrexed sodium hydrate), and pembrolizmab.

Muhammad Furqan: This will be determined based on data. The CheckMate trial 816 supports the use of nivolumab with platinum-doublet therapy.

Satoshi Watanabe: I think we have to choose treatment regimens based on the biomarkers including PD-L1 status. Single-agent immunotherapy is preferred for patients with high TPS and chemoimmunotherapy should be selected for patients with low-to-intermediate expression of PD-L1.

Stefano Bongiolatti: The results from the initial experiences with chemotherapy associated with ICIs are encouraging and promising because the MPR and $\mathrm{pCR}$ rates are higher than those reported by traditional chemotherapy and single-agent immunotherapy. In my opinion, when more data about DFS or OS are available, the combined approach between chemotherapy and immunotherapy could be advantageous for patients.

Yusuke Tomita: Immune checkpoint blockade combined with chemotherapy might be preferred because some clinical trials of immunotherapy combined with chemotherapy show higher a MPR rate compared to singleagent neoadjuvant immunotherapy. Immune checkpoint blockade combined with chemotherapy may be able to reduce a risk of hyperprogressive disease.

Filippo Longo: We do not perform neoadjuvant immunotherapy.

In-Jae Oh: I have no idea about the specific I-O regimen yet. But, early reports of global clinical trials show neoadjuvant nivolumab and atezolizumab are promising. A Chemo-I-O combination regimen does have not enough evidence.

Nobuhiko Seki: As the neoadjuvant immunotherapy, I expect to use immunotherapy combined with chemotherapy on the basis of a higher MPR rate compared to singleagent neoadjuvant immunotherapy. Furthermore, regarding immunotherapy in combination, I prefer the PD-1 inhibitors rather than the PD-L1 inhibitors according to the results of several phase II trials so far although I do not know for sure until the results of the phase III trials are available.

Pierfilippo Crucitti: We do not perform neoadjuvant immunotherapy.

Sai Yendamuri: [We prefer] chemoimmunotherapy.

Shinji Sasada: Numerous clinical trials have been conducted, but I am not sure which drug is better. If the patient's general condition is good, chemotherapy combination is preferred.

Ben G. L. Vanneste: [We prefer] a combination of nivolumab and ipilimumab because Cascone et al. (33). reported on this combination with MPR rates of $50 \%(8 / 16)$, compared with nivolumab alone. Nivolumab + ipilimumab resulted in higher pathologic complete response rates (10\% vs. $38 \%$ ), less viable tumor (median $50 \%$ vs. $9 \%$ ), and greater frequencies of effector, tissue-resident memory, and effector memory T cells.

Dirk De Ruysscher: PD-L1 $\leq 50 \%$ : in studies, but any anti-PD-1/L1 will be oaky when given with concurrent chemotherapy (squamous: carboplatin-paclitaxel; nonsquamous histology: carboplatin-pemetrexed). PD-L1 $>50 \%$ : in studies, but any anti-PD-1/L1 will do.

\section{(V) Do you think the surgery after neoadjuvant immunotherapy is more challenging? Why?}

Federico Cappuzzo: No, recent data are showing no increased risk (ASCO 2021).

Junji Uchino: Changes in the nature of the target region and fibrosis of the background lung, etc.

Kye Young Lee: It can be possible, but usually it is limited. Generally, I do not think that neoadjuvant immunotherapy involves significant difficulties for surgical techniques.

Mariano Provencio: No, I believe it has the same or similar difficulty as after chemotherapy and the published studies (NADIM and CM 816) do not indicate more difficulty, time, or complications than exclusive chemotherapy.

Paul Hofman: It seems according to some recent presentations made at the ASCO meeting (June 2021) that surgery is no more challenging after neoadjuvant immunotherapy. However some investigators previously 
reported difficulties due to lymph node inflammation response and also mediastinal fibrosis and/or some necrotic areas. The main challenge can be a risk of tumor progression between the initial diagnosis to the surgical decision due to the delay compared to the standard of care.

Takeo Nakada: Yes, I do. After neoadjuvant immunotherapy, inflammatory changes cause strong adhesions between the pulmonary arteries and metastatic lymph nodes. This may increase the risk of massive intraoperative bleeding.

Toyoaki Hida: No, according to the results of the CheckMate 816 study.

William Chi-Shing Cho: In general, after neoadjuvant immunotherapy, the surgical difficulty is increased, due to tissue proliferation, pulmonary fibrosis, and edema. The possible technical challenges during surgery and drug adverse effects during or after treatment, such as pneumonia and endocrinopathy, are still worthy of attention. However, we have a small amount of existing data showing that it is similar to other postinduction cases (postchemotherapy or postchemoradiotherapy). I think the CheckMate 816 shows that surgery after immunochemotherapy is not more complicated.

Haruhiko Sugimura: irAE, definitely.

Muhammad Furqan: I do not think so; however, it may be challenging in some cases due to inflammatory or fibrotic changes in tumor. The CheckMate 816 data do not suggest this is a concern.

Satoshi Watanabe: Yes. Neoadjuvant immunotherapy may increase the risk of postoperative complications.

Stefano Bongiolatti: Surgery after neoadjuvant immunotherapy is more challenging due to presence a strong scar tissue in the bronchovascular space which can lead to more extensive (pneumonectomies) or more complex (single or double sleeve) lung resection, sometimes in absence of a direct tumor invasion. This last issue could expose the patients to the risks and complications of an extended or a more complex procedure in the absence of cancer, but unfortunately, we do not have any data on the preoperative radiological imaging, and also the RECIST criteria are not completely reliable in this setting. In my opinion, if a surgeon is confident with lung resection after traditional neoadjuvant treatment, he/she may be initially surprised by the hilar scar tissue, but he/she could overcome this issue with his/her adequate technical background, keeping in mind that pneumonectomy after neoadjuvant treatment is not recommended due to the potential risk of severe complications and to the functional impairment that could preclude any adjuvant treatment.

Yusuke Tomita: There is no evidence to answer this question.

Filippo Longo: In my personal experience immunochemotherapy-treated patients develop tissue imbibition which makes surgery more complex (increased bleeding mainly because of a more fragile parenchyma). I am talking about patients who were initially judged not to be resectable for NSCLC but with a very good response to therapy that makes surgery feasible even months after an initial evaluation. Postoperative air leaks and significant persistent pleural effusion have been observed.

In-Jae Oh: Korean surgeons indicate that there is no difficulty after neoadjuvant I-O. But there are several difficulties, such as radiation fibrosis and adhesion after neoadjuvant (chemo)radiation. Personally, I worry about postoperative interstitial pneumonitis in case of neoadjuvant I-O especially in patients with pulmonary fibrosis or severe emphysema.

Nobuhiko Seki: I do not think the surgery after the neoadjuvant immunotherapy is more challenging because it does not seem to affect the morbidity and the early mortality significantly although the neoadjuvant immunotherapy may destroy the primary tumor vascularization and microenvironment, resulting in adhesions and fibrosis, increasing the difficulty and duration of surgery.

Pierfilippo Crucitti: In our experience, immunochemotherapytreated patients develop tissue imbibition which makes surgery more complex and there is an increased risk of major bleeding. Patients who are initially judged not resectable for NSCLC but with a very good response to therapy thus making surgery feasible even months after an initial evaluation being operated with a major incidence of postoperative air leaks as well as significant persistent pleural effusion has been observed.

Sai Yendamuri: Yes, surgery after neoadjuvant immunotherapy can be more challenging, due to the increased fibrosis but not always, and attempt to resect minimally invasively must be first made. However, the surgeon should be prepared to open.

Sang-Won Um: I do not think so. The difficulty of surgery after neoadjuvant immunotherapy or immunochemotherapy seems to be similar to that of surgery after neoadjuvant chemoradiation.

Shinji Sasada: I think that neoadjuvant immunotherapy may be similar to or advantageous to conventional cytotoxic chemotherapy in the absence of myelosuppression.

Ben G. L. Vanneste: Yes, there is evidence that neoadjuvant immunotherapy increases surgery-related complications; however, the texture of surrounding lungs 
and blood vessels and other tissues change. Caution is needed for additional surgical risks.

\section{(VI) Which condition is the most challenging surgery: neoadjuvant chemotherapy, neoadjuvant immunochemotherapy, or neoadjuvant tyrosine kinase inbibitor (TKI) therapy? Why?}

Federico Cappuzzo: The only data we have are with neoadjuvant chemotherapy, and it seems that the risk for complications is higher among patients receiving neoadjuvant.

Junji Uchino: I think it is neoadjuvant immunochemotherapy. It is difficult to judge the effect by the treatment, and identifying the excision region is also difficult. And, the lowering of the operative tolerance by AE such as ILD is also concerned.

Kye Young Lee: Maybe neoadjuvant immunochemotherapy, because treatment-related fibrosis in peritumoral tissues may provoke surgical complications.

Mariano Provencio: I believe that the most important thing in surgery after neoadjuvant is to achieve R0, and this is achieved in a high percentage [of patients] with the use of chemoimmunotherapy, so my preferences go that way.

Paul Hofman: I have no personal experience of the comparative impact of these regimens on surgery. Moreover, [I am] not sure that TKI therapy has a higher negative impact on surgery than does neoadjuvant immunotherapy or chemotherapy. The main challenge again is certainly the risk of tumor progression before surgery due to the delay.

Takeo Nakada: I think that neoadjuvant immunochemotherapy causes the most prominent inflammatory changes around the hilum. Neoadjuvant immunochemotherapy is the most challenging surgery.

Toyoaki Hida: Neoadjuvant chemotherapy (when anticancer drugs are not effective). Neoadjuvant TKI therapy (when TKIs are not effective, and when there is a risk of developing interstitial pneumonia as a side effect).

William Chi-Shing Cho: I think it is neoadjuvant TKI therapy. Nevertheless, there are many factors at play, such as the optimum neoadjuvant dosage, the optimal number of cycles, and the interval from the final administration of neoadjuvant agents to operation. We do not have largescale data on these issues. It is very important to study this carefully in ongoing and future trials. Sometimes, it depends on how long the patient receives treatment. For example, in an ongoing trial with 2-4 cycles of induction therapy, some cases are fairly simple. The challenge is that we are not very clear about the factors in predicting more difficult operations, especially due to the lack of surgical treatment experience for neoadjuvant immunochemotherapy with inflammatory effects, such as pneumonitis and endocrinopathies. Therefore, new biomarkers that can predict residual disease after neoadjuvant therapy are essential for selecting patients and improving clinical outcomes of treatment, including surgery. Along this line, the development of liquid biopsy (such as ctDNA) may help us avoid invasive restaging and further inform us of the utility of surgery when pCR occurs.

Haruhiko Sugimura: Again irAEs. Cooperative work with surgeon, oncologists and pathologists are necessary, but sometimes not well organized.

Muhammad Furqan: [It is] hard to answer this question without data. It is plausible that immunotherapy may cause more inflammatory and fibrotic changes compared to chemotherapy or TKI; however, the CheckMate 816 data support that the surgical outcomes are not different when compared to neoadjuvant chemotherapy.

Satoshi Watanabe: I think neoadjuvant immunochemotherapy has more risks of postoperative complications because patients would have AEs due to chemotherapy and irAEs due to immunotherapy.

Stefano Bongiolatti: I think it is very difficult to answer this question, because patients have different histories, different reactions, and different responses to the treatments. An accurate preoperative evaluation that includes all the patients' CT scans (from the beginning of the treatment) is the key to planning surgery in the safest and most effective way.

Yusuke Tomita: We need more evidence to answer this question.

Filippo Longo: In our experience radiochemotherapy is the standard approach in a neoadjuvant setting; subsequent surgery is always challenging because of anatomic remodeling and pleural adhesions, especially when minimally invasive procedures (VATS) are performed. I believe that major lung resections after immunochemotherapy is challenging as well mainly because a typically more fragile lung parenchyma.

In-Jae Oh: My though is neoadjuvant chemo is the most challenging surgery because the objective response rate (ORR) is about $30 \%$. But, the response rates of immunochemotherapy and TKI therapy are better than chemotherapy especially in patients with high PDL1 expression or driver mutation. Many surgeons and physicians want to improve after neoadjuvant treatment.

Nobuhiko Seki: Regarding the comparison between the neoadjuvant chemotherapy and the neoadjuvant 
immunochemotherapy, I do not think there exists a difference in surgical difficulty. In AACR2021 and ASCO2021, nivolumab + platinum-doublet chemotherapy $v s$. chemotherapy as neoadjuvant treatment for resectable (IB-IIIA) NSCLC in the phase 3 CheckMate 816 trial was reported. In this trial, surgery-related SEs indicated no difference between the groups. On the other hand, because there are no controlled trials so far, I do not have a clear answer regarding the comparison between neoadjuvant chemotherapy and neoadjuvant TKI therapy. At present, the phase 3 trial of osimertinib with or without chemotherapy $v s$. chemotherapy alone as neoadjuvant therapy for patients with EGFR mutation-positive resectable NSCLC (NeoADAURA) is ongoing. Therefore, I think we should wait for the results of this trial to conclude if there exists a difference in surgical difficulty between the groups.

Pierfilippo Crucitti: In our experience radiochemotherapy is the standard approach in a neoadjuvant setting; subsequent surgery is always challenging because of anatomic remodeling and pleural adhesions, especially when minimally invasive procedures (VATS) are performed. I believe that major lung resections after immunochemotherapy are also more challenging mainly because the lung parenchyma is more fragile.

Sai Yendamuri: Neoadjuvant immunochemotherapy [is more challenging] due to the issues outlined above.

Sang-Won Um: Surgery after neoadjuvant immunochemotherapy seems to the most challenging since neoadjuvant immunochemotherapy may cause adhesion and fibrosis of mediastinal or hilar structures.

Shinji Sasada: I think neoadjuvant immunochemotherapy will be the most challenging of surgery. This is because it is necessary to consider immunosuppression by cytotoxic chemotherapy and irAEs by immunotherapy. It is also likely that immunotherapy will cause local fibrosis.

Dirk De Ruysscher: Neoadjuvant treatment with immunotherapy [is the most challenging] because of fibrosis.

\section{Acknowledgments}

Funding: None.

\section{Footnote}

Conflicts of Interest: All authors have completed the ICMJE uniform disclosure form (available at https://dx.doi. org/10.21037/tlcr-21-634). Dr. IJO has grant from Roche; he also has received consulting fee from Roche, Ono, MSD, Pfizer, Boehringer-Ingelheim, AstraZeneca and Takeda. Dr. YT reports grants from JSPS KAKENHI grant number JP18K15928 and grants from Takeda Science Foundation outside the submitted work. Dr. SW reports grant and personal fee from AstraZeneca and Boehringer Ingelheim, personal fee from Chugai Pharma, Ono Pharmaceutical, Bristol-Myers, Eli Lilly, MSD, Taiho Pharmaceutical, Pfizer, Novartis, Daiichi Sankyo. Dr. RC has received consulting fees, Payment or honoraria for lectures, presentations, speakers bureaus, manuscript writing or educational events from AZ, MSD, BMS, Roche, TAKEDA, Pfizer, janssen, Bayer, Novartis; Dr. RC has Participation on a Data Safety Monitoring Board or Advisory Board with AZ, MSD, BMS, Roche, TAKEDA, Pfizer, janssen, Bayer, Novartis; Dr. RC has received Support for attending meetings and/ or travel from Roche, MSD, Takeda; Dr. RC declares stock ownership with The christie private care. Dr. KM reports medical education grant, Africa from Varian and Pfizer, Clinical trial grant from AstraZeneca and Novartis; travel and accommodations to a clinical trial meeting, 2019 from AstraZeneca; Dr. KM is an unpaid Board of Directors of Global Access to Cancer Care Foundation. Dr. JCS has grant support from National Institute of Health and the Noah's Hope Foundation; she also has a family member that owns stock options and receives financial compensation from Vida Diagnostics. Dr. MF participated in advisory board for Astrazeneca, AbbVie and Beigene LLC. Dr. DDR reports grants from Bristol-Myers Squibb, grants from AstraZeneca, grants from Boehringer Ingelheim, from Philips, from Olink, from Celgene, from Seattle Genetics, from Roche/Genentech, from Merck/Pfizer, outside the submitted work. Dr. FC reports fees for membership of an advisory board from Roche, AstraZeneca, BMS, Pfizer, Takeda, Lilly, Bayer, Amgen, Sanofi, Pharmamar, Novocure and MSD. Dr NS obtained commercial research grants from Eli Lilly, Chugai Pharmaceutical, Taiho Pharmaceutical, Pfizer Japan, Ono Pharmaceutical, and Nippon Boehringer Ingelheim, and has received speaking honoraria from Eli Lilly, AstraZeneca, MSD Oncology, Chugai Pharmaceutical, Taiho Pharmaceutical, Pfizer Japan, Ono Pharmaceutical, Nippon Boehringer Ingelheim, and Bristol-Myers Squibb Japan. TH serves as an unpaid editorial board member of Translational Lung Cancer Research from Jan 2020 to Dec 2021. FC serves as an unpaid editorial board member of Translational Lung Cancer Research from Aug 2021 to Jul 2023. MF serves as an unpaid editorial board member of Translational Lung Cancer Research from 
Sep 2019 to Sep 2021. SY serves as an unpaid editorial board member of Translational Lung Cancer Research from Sep 2019 to Sep 2021. GM serves as an unpaid editorial board member of Translational Lung Cancer Research from Aug 2021 to Jul 2023. PH serves as an unpaid editorial board member of Translational Lung Cancer Research from Mar 2021 to Feb 2023. The other authors have no conflicts of interest to declare.

Ethical Statement: The authors are accountable for all aspects of the work in ensuring that questions related to the accuracy or integrity of any part of the work are appropriately investigated and resolved.

Open Access Statement: This is an Open Access article distributed in accordance with the Creative Commons Attribution-NonCommercial-NoDerivs 4.0 International License (CC BY-NC-ND 4.0), which permits the noncommercial replication and distribution of the article with the strict proviso that no changes or edits are made and the original work is properly cited (including links to both the formal publication through the relevant DOI and the license). See: https://creativecommons.org/licenses/by-nc-nd/4.0/.

\section{References}

1. Chen $\mathrm{W}$, Zheng R, Baade PD, et al. Cancer statistics in China, 2015. CA Cancer J Clin 2016;66:115-32.

2. Erratum: Global cancer statistics 2018: GLOBOCAN estimates of incidence and mortality worldwide for 36 cancers in 185 countries. CA Cancer J Clin 2020;70:313.

3. Eberhardt WE, Mitchell A, Crowley J, et al. The IASLC Lung Cancer Staging Project: Proposals for the Revision of the M Descriptors in the Forthcoming Eighth Edition of the TNM Classification of Lung Cancer. J Thorac Oncol 2015;10:1515-22.

4. Rusch VW, Chansky K, Kindler HL, et al. The IASLC Mesothelioma Staging Project: Proposals for the M Descriptors and for Revision of the TNM Stage Groupings in the Forthcoming (Eighth) Edition of the TNM Classification for Mesothelioma. J Thorac Oncol 2016;11:2112-9.

5. Horita N, Miyazawa N, Morita S, et al. Preoperative chemotherapy is effective for stage III resectable non-small-cell lung cancer: metaanalysis of 16 trials. Clin Lung Cancer 2013;14:488-94.

6. Pankova OV, Rodionov EO, Miller SV, et al. Neoadjuvant chemotherapy combined with intraoperative radiotherapy is effective to prevent recurrence in high-risk non-small cell lung cancer (NSCLC) patients. Transl Lung Cancer Res 2020;9:988-99.

7. Blank CU, Rozeman EA, Fanchi LF, et al. Neoadjuvant versus adjuvant ipilimumab plus nivolumab in macroscopic stage III melanoma. Nat Med 2018;24:1655-61.

8. Cloughesy TF, Mochizuki AY, Orpilla JR, et al. Neoadjuvant anti-PD-1 immunotherapy promotes a survival benefit with intratumoral and systemic immune responses in recurrent glioblastoma. Nat Med 2019;25:477-86.

9. Forde PM, Chaft JE, Smith KN, et al. Neoadjuvant PD-1 blockade in resectable lung cancer. $\mathrm{N}$ Engl J Med 2018;378:1976-86.

10. Liang W, Cai K, Chen C, et al. Expert consensus on neoadjuvant immunotherapy for non-small cell lung cancer. Transl Lung Cancer Res 2020;9:2696-715.

11. Goldstraw P, Chansky K, Crowley J, et al. The IASLC Lung Cancer Staging Project: Proposals for Revision of the TNM Stage Groupings in the Forthcoming (Eighth) Edition of the TNM Classification for Lung Cancer. J Thorac Oncol 2016;11:39-51.

12. Park BJ, Cho JH, Lee JH, et al. Temporal and regional distribution of initial recurrence site in completely resected N1-stage II lung adenocarcinoma: The effect of postoperative adjuvant chemotherapy. Lung Cancer 2018;117:7-13.

13. Marra A, Hillejan L, Zaboura G, et al. Pathologic N1 non-small cell lung cancer: correlation between pattern of lymphatic spread and prognosis. J Thorac Cardiovasc Surg 2003;125:543-53.

14. Boyd JA, Hubbs JL, Kim DW, et al. Timing of local and distant failure in resected lung cancer: implications for reported rates of local failure. J Thorac Oncol 2010;5:211-4.

15. Lu S, Wang J, Yu Y, et al. Tislelizumab plus chemotherapy as first-line treatment for locally advanced or metastatic nonsquamous NSCLC (RATIONALE 304): a randomized phase 3 trial. J Thorac Oncol 2021;16:1512-22.

16. Antonia SJ, Balmanoukian A, Brahmer J, et al. Clinical activity, tolerability, and long-term follow-up of durvalumab in patients with advanced NSCLC. J Thorac Oncol 2019;14:1794-806.

17. Borghaei H, Paz-Ares L, Horn L, et al. Nivolumab versus docetaxel in advanced nonsquamous non-small-cell lung cancer. N Engl J Med 2015;373:1627-39.

18. Coffey JC, Wang JH, Smith MJ, et al. Excisional surgery for cancer cure: therapy at a cost. Lancet Oncol 2003;4:760-8. 
19. Xu P, Zhang P, Sun Z, et al. Surgical trauma induces postoperative $T$-cell dysfunction in lung cancer patients through the programmed death-1 pathway. Cancer Immunol Immunother 2015;64:1383-92.

20. Liu J, Blake SJ, Yong MC, et al. Improved efficacy of neoadjuvant compared to adjuvant immunotherapy to eradicate metastatic disease. Cancer Discov 2016;6:1382-99.

21. Wu SP, Liao RQ, Tu HY, et al. Stromal PD-L1-positive regulatory $\mathrm{T}$ cells and $\mathrm{PD}-1$-positive CD8-positive t cells define the response of different subsets of non-small cell lung cancer to PD-1/PD-L1 blockade immunotherapy. J Thorac Oncol 2018;13:521-32.

22. Anagnostou V, Forde PM, White JR, et al. Dynamics of tumor and immune responses during immune checkpoint blockade in non-small cell lung cancer. Cancer Res 2019;79:1214-25.

23. Herbst RS, Baas P, Kim DW, et al. Pembrolizumab versus docetaxel for previously treated, PD-L1-positive, advanced non-small-cell lung cancer (KEYNOTE-010): a randomised controlled trial. Lancet 2016;387:1540-50.

24. Reck M, Rodríguez-Abreu D, Robinson AG, et al. Pembrolizumab versus chemotherapy for PD-L1-positive non-small-cell lung cancer. N Engl J Med 2016;375:1823-33.

25. Ettinger DS, Wood DE, Aggarwal C, et al. NCCN guidelines insights: non-small cell lung cancer, version 1.2020. J Natl Compr Canc Netw 2019;17:1464-72.

26. Dong ZY, Zhang JT, Liu SY, et al. EGFR mutation correlates with uninflamed phenotype and weak immunogenicity, causing impaired response to PD-1 blockade in non-small cell lung cancer. Oncoimmunology 2017;6:e1356145.

27. Azuma K, Ota K, Kawahara A, et al. Association of PDL1 overexpression with activating EGFR mutations in surgically resected nonsmall-cell lung cancer. Ann Oncol 2014;25:1935-40.

28. Dong ZY, Zhong WZ, Zhang XC, et al. Potential predictive value of TP53 and KRAS mutation status for response to PD-1 blockade immunotherapy in lung adenocarcinoma. Clin Cancer Res 2017;23:3012-24.

29. Eichhorn F, Kriegsmann M, Klotz LV, et al. Prognostic impact of PD-L1 expression in pN1 NSCLC: a retrospective single-center analysis. Cancers (Basel) 2021;13:2046.

30. Rusch V, Chaft J, Johnson B, et al. MA04.09 Neoadjuvant Atezolizumab in resectable non-small cell lung cancer (NSCLC): updated results from a multicenter study (LCMC3). J Thorac Oncol 2018;13:S369.

31. Wu YL, Yang XN, Zhong W, et al. Multi-centre randomized controlled study comparing adjuvant vs neoadjuvant chemotherapy with docetaxel plus carboplatin in resectable stage IB to IIIA NSCLC: final results of CSLC0501. Ann Oncol 2016;27:vi407.

32. Chaft JE, Rusch V, Ginsberg MS, et al. Phase II trial of neoadjuvant bevacizumab plus chemotherapy and adjuvant bevacizumab in patients with resectable nonsquamous nonsmall-cell lung cancers. J Thorac Oncol 2013;8:1084-90.

33. Cascone T, William WN Jr, Weissferdt A, et al. Neoadjuvant nivolumab or nivolumab plus ipilimumab in operable non-small cell lung cancer: the phase 2 randomized NEOSTAR trial. Nat Med 2021;27:504-14.

34. Shu CA, Gainor JF, Awad MM, et al. Neoadjuvant atezolizumab and chemotherapy in patients with resectable non-small-cell lung cancer: an open-label, multicentre, single-arm, phase 2 trial. Lancet Oncol 2020;21:786-95.

35. Rothschild SI, Zippelius A, Eboulet EI, et al. 1237MO SAKK 16/14: Anti-PD-L1 antibody durvalumab in addition to neoadjuvant chemotherapy in patients with stage IIIA (N2) non-small cell lung cancer (NSCLC)-A multicenter single-arm phase II trial. Ann Oncol 2020;31:S803-S4.

36. Provencio M, Nadal E, Insa A, et al. Neoadjuvant chemotherapy and nivolumab in resectable non-small-cell lung cancer (NADIM): an open-label, multicentre, singlearm, phase 2 trial. Lancet Oncol 2020;21:1413-22.

37. Yang CJ, McSherry F, Mayne NR, et al. Surgical outcomes after neoadjuvant chemotherapy and ipilimumab for nonsmall cell lung cancer. Ann Thorac Surg 2018;105:924-9.

38. Rijavec E, Indini A, Ghidini M, et al. Nivolumab plus ipilimumab for the first-line treatment of metastatic NSCLC. Expert Rev Anticancer Ther 2021;21:705-13.

39. Reuss JE, Anagnostou V, Cottrell TR, et al. Neoadjuvant nivolumab plus ipilimumab in resectable non-small cell lung cancer. J Immunother Cancer 2020;8:e001282.

40. Larkin J, Chiarion-Sileni V, Gonzalez R, et al. Combined nivolumab and ipilimumab or monotherapy in untreated melanoma. N Engl J Med 2015;373:23-34.

41. Hong MH, Ahn B, Kim HR, et al. FP03.02 Interim analysis of neoadjuvant chemoradiotherapy and durvalumab for potentially resectable stage III non-small cell lung cancer (NSCLC). J Thorac Oncol 2021;16:S194-5.

42. Zhou C, Wang Y, Zhao J, et al. Efficacy and biomarker analysis of camrelizumab in combination with apatinib in patients with advanced nonsquamous NSCLC previously treated with chemotherapy. Clin Cancer Res 2021;27:1296-304.

43. Travis WD, Dacic S, Wistuba I, et al. IASLC 
multidisciplinary recommendations for pathologic assessment of lung cancer resection specimens after neoadjuvant therapy. J Thorac Oncol 2020;15:709-40.

44. William WN Jr, Pataer A, Kalhor N, et al. Computed tomography RECIST assessment of histopathologic response and prediction of survival in patients with resectable non-small-cell lung cancer after neoadjuvant chemotherapy. J Thorac Oncol 2013;8:222-8.

45. Apalla Z, Papageorgiou C, Lallas A, et al. Cutaneous adverse events of immune checkpoint inhibitors: a literature review. Dermatol Pract Concept 2021;11:e2021155.

46. Ferrara R, Mezquita L, Texier M, et al. Hyperprogressive disease in patients with advanced non-small cell lung cancer treated with PD-1/PD-L1 inhibitors or with singleagent chemotherapy. JAMA Oncol 2018;4:1543-52.

47. Lee JH, Long GV, Menzies AM, et al. Association between circulating tumor DNA and pseudoprogression in patients with metastatic melanoma treated with anti-programmed cell death 1 antibodies. JAMA Oncol 2018;4:717-21.

48. Ma Y, Wang Q, Dong Q, et al. How to differentiate pseudoprogression from true progression in cancer patients treated with immunotherapy. Am J Cancer Res 2019;9:1546-53.

49. Vekens K, Everaert H, Neyns B, et al. The value of 18F-FDG PET/CT in predicting the response to PD-1 blocking immunotherapy in advanced NSCLC patients with high-level PD-L1 expression. Clin Lung Cancer 2021. [Epub ahead of print]. doi: 10.1016/ j.cllc.2021.03.001.

50. Junker K, Langner K, Klinke F, et al. Grading of tumor regression in non-small cell lung cancer : morphology and prognosis. Chest 2001;120:1584-91.

51. Pataer A, Kalhor N, Correa AM, et al. Histopathologic response criteria predict survival of patients with resected lung cancer after neoadjuvant chemotherapy. J Thorac Oncol 2012;7:825-32.

52. Hellmann MD, Chaft JE, William WN Jr, et al. Pathological response after neoadjuvant chemotherapy in resectable non-small-cell lung cancers: proposal for the use of major pathological response as a surrogate endpoint. Lancet Oncol 2014;15:e42-50.

53. Chaft JE, Hellmann MD, Velez MJ, et al. Initial experience with lung cancer resection after treatment with T-cell checkpoint inhibitors. Ann Thorac Surg 2017;104:e217-8.

54. Bott MJ, Yang SC, Park BJ, et al. Initial results of pulmonary resection after neoadjuvant nivolumab in patients with resectable non-small cell lung cancer. J
Thorac Cardiovasc Surg 2019;158:269-76.

55. Bott MJ, Cools-Lartigue J, Tan KS, et al. Safety and feasibility of lung resection after immunotherapy for metastatic or unresectable tumors. Ann Thorac Surg 2018;106:178-83.

56. Yang CF, Adil SM, Anderson KL, et al. Impact of patient selection and treatment strategies on outcomes after lobectomy for biopsy-proven stage IIIA pN2 non-small cell lung cancer. Eur J Cardiothorac Surg 2016;49:1607-13.

57. Krantz SB, Mitzman B, Lutfi W, et al. Neoadjuvant chemoradiation shows no survival advantage to chemotherapy alone in stage IIIA patients. Ann Thorac Surg 2018;105:1008-16.

58. Liang H, Yang C, Gonzalez-Rivas D, et al. Sleeve lobectomy after neoadjuvant chemoimmunotherapy/ chemotherapy for local advanced non-small cell lung cancer. Transl Lung Cancer Res 2021;10:143-55.

59. NSCLC Meta-analysis Collaborative Group. Preoperative chemotherapy for non-small-cell lung cancer: a systematic review and meta-analysis of individual participant data. Lancet 2014;383:1561-71.

60. Gandhi L, Rodríguez-Abreu D, Gadgeel S, et al. Pembrolizumab plus chemotherapy in metastatic nonsmall-cell lung cancer. N Engl J Med 2018;378:2078-92.

61. Wang DY, Salem JE, Cohen JV, et al. Fatal toxic effects associated with immune checkpoint inhibitors: a systematic review and meta-analysis. JAMA Oncol 2018;4:1721-8.

62. Liu J, O'Donnell JS, Yan J, et al. Timing of neoadjuvant immunotherapy in relation to surgery is crucial for outcome. Oncoimmunology 2019;8:e1581530.

Cite this article as: Qiu B, Cai K, Chen C, Chen J, Chen KN, Chen QX, Cheng C, Dai TY, Fan J, Fan Z, Hu J, Hu WD, Huang YC, Jiang GN, Jiang J, Jiang T, Jiao WJ, Li HC, Li Q, Liao YD, Liu HX, Liu JF, Liu L, Liu Y, Long H, Luo QQ, Ma HT, Mao NQ, Pan XJ, Tan F, Tan LJ, Tian H, Wang D, Wang WX, Wei L, Wu N, Wu QC, Xiang J, Xu SD, Yang L, Zhang H, Zhang L, Zhang P, Zhang Y, Zhang Z, Zhu K, Zhu Y, Um SW, Oh IJ, Tomita Y, Watanabe S, Nakada T, Seki N, Hida T, Sasada S, Uchino J, Sugimura H, Dermime S, Cappuzzo F, Rizzo S, Cho WCS, Crucitti P, Longo F, Lee KY, De Ruysscher D, Vanneste BGL, Furqan M, Sieren JC, Yendamuri S, Merrell KW, Molina JR, Metro G, Califano R, Bongiolatti S, Provencio M, Hofman P, Gao S, He J. Expert consensus on perioperative immunotherapy for local advanced non-small cell lung cancer. Transl Lung Cancer Res 2021;10(9):3713-3736. doi: 10.21037/tlcr-21-634 